\title{
Die Akzeptanz von Flüchtlingen. Eine vergleichende Studie sechs deutscher Wohngebiete
}

\section{The acceptance of refugees. A comparative study of six German neighbourhoods}

https://doi.org/10.2478/rara-2019-0015

Eingegangen: 7. Juni 2018; Angenommen: 6. Februar 2019

Zusammenfassung: In zahlreichen Wohngebieten deutscher Städte sind seit 2011 Wohnunterkünfte für Flüchtlinge errichtet worden. Das war häufig sehr umstritten, unter anderem wegen mangelnder Beteiligung der Bürger und der Größe der Einrichtungen. Der vorliegende Beitrag untersucht die Einstellungen der Bewohner von Wohngebieten mit Flüchtlingsunterkünften sowohl zu den Flüchtlingen als auch zu den Unterkünften. In drei Städten (Hamburg, Köln und Mülheim an der Ruhr) wurden in jeweils zwei Wohngebieten repräsentative Stichproben gezogen und insgesamt 1.800 Anwohner befragt. Die theoretische Grundlage bilden die Vorurteilstheorie und die Kontakt-Hypothese. Wir untersuchen die Effekte der Struktur des Wohngebietes (unter anderem soziale Zusammensetzung, kollektive Effektivität) und individueller Merkmale auf die Einstellungen. Die Einstellungen gegenüber den Flüchtlingen und den Unterkünften sind sehr differenziert. Mit steigender Bildung der Individuen und steigendem Status des Wohngebiets sind sie sowohl zu den Flüchtlingen als auch zu der Einrichtung positiver. Kontakte zu Flüchtlingen sind mit geringeren Vorurteilen verbunden, mit weniger Ängsten gegenüber Flüchtlingen und einer höheren Toleranz gegenüber einem höheren Anteil von Flüchtlingen im Wohngebiet. Damit bewähren sich sowohl die Hypothesen der Vorurteilstheorie, als auch die Kontakt-Hypothese. Wir erörtern abschließend unter welchen Bedingungen die Unterbringung von Flüchtlingen in Wohngebieten sozial verträglich ist.

Schlüsselwörter: Migration; Integration; Flüchtlinge; Kontakt-Hypothese; Wohngebiete

Abstract: In almost all German cities since 2011 accommodations for refugees were established in residential areas, often leading to protest among the residents of respective neighbourhoods. Our study examines the attitudes of residents towards refugees and accommodations for them in their housing area. We conducted face-to-face interviews with representative samples of residents in six such neighbourhoods in Hamburg, Cologne, and Mülheim an der Ruhr, Germany, and obtained a total of 1,800 interviews. Based on hypotheses from prejudice theory and the contact hypothesis we study effects of the social status of the neighbourhood, neighbourhood social capital (collective efficacy), and individual characteristics on refugee discrimination. We find attitudes towards refugees and their accommodations to be predominantly positive, although differentiated in several ways. Discrimination is lower in higher social status neighbourhoods and decreases with years of schooling. Further, contact to refugees

\footnotetext{
*Corresponding author: Felix Leßke, M.A., Universität zu Köln, Institut für Soziologie und Sozialpsychologie, Universitätsstraße 77, 50931 Köln, Deutschland, E-mail: lesske@wiso.uni-koeln.de

Prof. Dr. Jürgen Friedrichs: Universität zu Köln, Institut für Soziologie und Sozialpsychologie, Universitätsstraße 77, 50931 Köln, Deutschland

Vera Schwarzenberg, M.A.: Universität zu Köln, Institut für Soziologie und Sozialpsychologie, Universitätsstraße 77, 50931 Köln, Deutschland

\#Jürgen Friedrichs ist leider noch während der Veröffentlichung dieses Schwerpunktheftes verstorben. Wir möchten die Gelegenheit nutzen, um unseren Dank für die lehrreiche und schöne Zeit der Zusammenarbeit auszudrücken.
} 
reduces prejudice and increases tolerance of larger shares of refugees in one's neighbourhood. In the final section we suggest, which type of neighbourhood is favourable for refugee accommodations.

Keywords: Migration; Integration; Refugees; Contact hypothesis; Neighbourhoods

\section{Problem}

Mit der seit 2011 steigenden Zahl der Flüchtlinge in Deutschland, insbesondere der außergewöhnlich hohen Zuwanderung im Jahr 2015, sind die Kommunen vor Probleme der Unterbringung und der Integration der Flüchtlinge gestellt worden, auf die sie nicht vorbereitet waren (Aumüller/Daphi/Biesenkamp 2015; Dymarz/Hanhörster/Hans et al. 2016; Aumüller 2018; Grote 2018). Das oberste Ziel war, die Flüchtlinge unterzubringen. Das geschah dann in Turnhallen, Leichtbauhallen, Wohnungen, neu errichteten Container-Siedlungen, Systembauten oder ehemaligen Bürogebäuden.

In vielen Städten wurde nach dem Grundsatz verfahren, die Unterkünfte über alle Wohngebiete der Stadt zu verteilen, also ungeachtet des sozialen Status, sondern primär nach Verfügbarkeit städtischer Flächen. Weil man die Unterkünfte dringend benötigte, um der Verpflichtung der öffentlich-rechtlichen Unterbringung nachzukommen, wurden in vielen Fällen die Bewohner eines Wohngebiets nicht oder nur unzureichend beteiligt (Bauer 2017). Die Bereitschaft, eine Flüchtlingsunterkunft in der Nachbarschaft zu akzeptieren, hängt laut Aumüller, Daphi und Biesenkamp (2015: 129 f.) nicht zuletzt vom politischen Beteiligungsprozess der Anwohner und der Art der Unterbringung (dezentral in Wohnungen vs. zentral in Unterkünften) ab. Noch gewichtiger jedoch sind folgende Bedingungen: die Größe der Einrichtung und der soziale Status der Bewohner des Gebiets. So konnten Lubbers, Coenders und Scheepers (2006: 248) für Befragte in niederländischen Städten zeigen, dass Unterkünfte mit 50 oder 100 Flüchtlingen eher akzeptiert werden als solche mit 500 Flüchtlingen - letztere wurden von $40 \%$ der Befragten abgelehnt (vgl. auch Aumüller/ Daphi/Biesenkamp 2015: 61). Schwieriger ist es, den Einfluss des sozialen Status der Bewohnerschaft auf die Toleranz nachzuweisen; hierzu fehlen empirische Studien. Wir können nur aus Studien über Vorurteile und Diskriminierung folgern, dass mit steigender Bildung und steigendem Einkommen auch die Toleranz zunimmt (vgl. Kapitel 2). Dann wären statushohe Wohngebiete besonders gut und statusniedrige besonders wenig geeignet, in innen Flüchtlingsunterkünfte zu errichten.

Ziel des Beitrags ist, die Einstellungen der Anwohner zu der jeweiligen Flüchtlingsunterkunft und den Flücht- lingen am Beispiel von sechs Wohngebieten (je zwei in Hamburg, Köln und Mülheim an der Ruhr) zu erfassen, zu beschreiben und zu erklären.

Hamburg wurde ausgewählt, weil dort als Stadtstaat ein einstufiges Unterbringungssystem vorhanden ist, Köln, da hier die dem Beitrag zugrunde liegende Studie angesiedelt ist und Mülheim an der Ruhr, weil hier die Flüchtlinge bis 2014 dezentral untergebracht wurden und es eine sehr medienwirksame Willkommenskultur gab. Wir stellen in diesem Aufsatz die Einstellungen der Anwohner dar. Für die Ergebnisse der Experteninterviews sei auf Friedrichs/Leßke/Schwarzenberg (2017) und Friedrichs/ Leßke/Schwarzenberg (2019) verwiesen, dort untersuchen wir die Effekte der strukturellen Bedingungen der Städte auf die Integrationschancen der Flüchtlinge.

Wir stellen zuerst die theoretischen Grundlagen, die Hypothesen und ihre Operationalisierung dar. Im zweiten Teil des Beitrags beschreiben wir die Untersuchungsgebiete, die Stichproben und Forschungsmethoden. Es folgt ein umfangreicher dritter Teil mit den Ergebnissen und dem Test der Hypothesen. Im Fazit erörtern wir die Ergebnisse im Hinblick auf die theoretischen Annahmen und machen Vorschläge für die (politische) Planung.

\section{Theorie}

Ausgangspunkt unserer Studie sind die Einstellungen der Bewohner in Wohngebieten, in denen Flüchtlinge untergebracht wurden, zu der Unterkunft und zu Flüchtlingen generell. Um die Einstellungen gegenüber Flüchtlingen zu untersuchen, beziehen wir uns auf Theorien der Vorurteilsforschung.

\subsection{Vorurteile und Diskriminierung}

Die klassische Theorie der Vorurteile stammt von Allport (1954). Ihr zufolge schreiben Angehörige der Majorität (ingroup, hier: Deutsche) Angehörigen der Minorität (outgroup, hier: Flüchtlinge) negative Eigenschaften zu, mit der Folge, dass Letztere diskriminiert werden (Allport 1954: 29 ff.; Quillian 1995; vgl. Baur/Klein/Seuring et al. 2010: 3; Leibold/Thörner/Gosen et al. 2012: 179). 
Zwei entscheidende Bedingungen für eine Diskriminierung sind die (wahrgenommenen) Anteile der Minorität an der Gesamtbevölkerung und die Kontakte zwischen Majorität und Minorität. Hier stehen sich zwei rivalisierende Hypothesen gegenüber: (1) Mit steigendem Anteil der Minorität erhöht sich die Konkurrenz um knappe Ressourcen und damit die Diskriminierung (Blalock 1967; Quillian 1995; Quillian 1996; Taylor 1998; Fetzer 2000; Taylor 2000). (2) Mit steigendem Anteil der Minorität sinkt die Diskriminierung, weil die Majorität ihr stärker ausgesetzt ist, dadurch steigen die Kontaktchancen und aufgrund der Kontakte verringern sich die Vorurteile und mithin die Diskriminierung (Schelling 1971). Zahlreiche Studien sowie der Überblick zur Kontakt-Hypothese von Pettigrew und Tropp $(2006$; 2011) belegen die zweite Hypothese (Wagner/Christ/Kühnel 2003; Mansel 2006: $97 \mathrm{f}$.; von Gostomski/Stichs/Stichs 2008; Farwick 2009: 142; Martinović/van Tubergen/Maas 2009; Schlueter/Scheepers 2010; Ellison/Shin/Leal 2011; Martinović 2013; Petermann/Schönwälder 2014; Schmid/Al Ramiah/Hewstone 2014). Wir untersuchen, welche der beiden Hypothesen sich für den verstärkten Zuzug von Flüchtlingen seit 2011 für die untersuchten Gebiete bewährt.

\subsection{Die Kontakt-Hypothese}

Die grundlegende Annahme der Kontakt-Hypothese ist, dass Kontakt zwischen Gruppenmitgliedern (hier: Deutsche und Flüchtlinge) zu Sympathie führt. Die KontaktHypothese ist in zahlreichen Studien untersucht worden. Systematische Übersichten über die Ergebnisse geben Pettigrew (1998), Pettigrew und Tropp (2006) sowie Pettigrew und Tropp (2011). Sie unterzogen 515 Studien mit 713 unabhängigen Stichproben einer Metaanalyse und gelangten zu dem Ergebnis, dass sich die Annahme, Kontakt führe zu Sympathie und beuge Vorurteilen vor, in fast allen Studien bewährt, doch bedarf es einiger zusätzlicher Bedingungen. Die Sympathie steigt, wenn a) es sich um statusgleiche Personen handelt, b) Wissen über die jeweils andere Gruppe sowie c) weniger Angst vor der fremden Gruppe besteht und d) Empathie aufgebaut wird, das heißt, man fähig ist, sich in die Lage und Einstellungen der anderen Gruppe hineinzuversetzen (Pettigrew/Tropp 2011: 77 f., 94 f.).

Der Kontakt-Hypothese liegt ein Prozess zugrunde, der in mehreren Phasen abläuft (Pettigrew 1998: 70 ff.): Kontakt $>$ Bessere Kenntnisse der Fremdgruppe $>$ Veränderte Einstellung („Dekategorisierung“) > Weitere Kontakte > Verändertes Verhalten gegenüber der Fremdgruppe $>$ Veränderte Bewertung der Fremdgruppe.
Von sehr ähnlichen Befunden berichten mehrere europäische Studien, die sich mit dem Zusammenhang von Anteil der Minorität, wirtschaftlichen Bedingungen, Diskriminierung und individuellen Merkmalen von Flüchtlingen in Wohngebieten befassen. Hier zeigt sich, dass nicht der objektive, sondern der subjektiv wahrgenommene Anteil der Minorität wichtig ist (Stephan/Ybarra/ Morrison 2009); er führt zu einer höheren wahrgenommenen Bedrohung und diskriminierenden Einstellungen (Scheepers/Gijsberts/Coenders 2002; Semyonov/ Raijman/Anat et al. 2004; Schneider 2008). Aufgrund der enormen medialen Berichterstattung, häufig mit negativ konnotierten Formulierungen, wie „Flüchtlingswelle“, könnte der subjektiv wahrgenommene Anteil der Flüchtlinge demnach diskriminierendes Verhalten begünstigen.

Mit Daten des European Social Survey konnten Billiet, Meuleman und de Witte (2014) zeigen, dass die Höhe des Bruttoinlandsprodukts negativ mit der wahrgenommenen Bedrohung korreliert und Personen mit niedriger Bildung, niedrigem Einkommen oder Arbeitslose sich stärker bedroht sehen.

Die Kontakt-Hypothese ist auch ein zentraler Bestandteil der bereits erwähnten Vorurteils-Theorie von Allport (1954: 26 f.; Baur/Klein/Seuring et al. 2010: 4; Asbrock/Kauff/Issmer et al. 2012: 200). Allport (1954: 281) schreibt dazu: „Prejudice (unless deeply rooted in the character structure of the individual) may be reduced by equal status contact between majority and minority groups in pursuit of common goals. The effect is greatly enhanced if this contact is sanctioned by institutional supports (i.e., by law, custom or local atmosphere), and provided it is of a sort that leads to the perception of common interests and common humanity between members of the two groups."

Von den im Zitat benannten vier Bedingungen („Sozialer Status der interagierenden Personen“; „Qualität der Kontakte“; "gemeinsame Ziele“; „institutionelle Unterstützung"), unter denen sich nach Allport (1954: 281) Vorurteile durch Kontakte abbauen lassen, erscheint uns besonders der vierte Punkt als gegeben. Die Begleitung der "Willkommenskultur" durch hauptamtliche Ehrenamtskoordinatoren ist hierfür ein Beispiel. Es gibt jedoch auch den entgegengesetzten Fall: Wenn in einzelnen Gemeinden ein Klima der Fremdenfeindlichkeit herrscht, dann werden Kontakte unterbunden und entsprechend die Vorurteile aufrechterhalten.

Liegen positive Bedingungen vor, führen Kontakte eher zu verringerten Vorurteilen (Wagner/Christ/Kühnel 2003; Hewstone 2004: 10; Dixon 2006). Smith (1994) fand heraus, dass jede einzelne Bedingung einen wenngleich geringen - Effekt darauf hat, die Vorurteile 
zu verringern. Auch indirekte Kontakte, z. B. von Familienmitgliedern, tragen dazu bei (Pettigrew/Tropp 2000; Hewstone 2004). Ebenso wie die Kontaktchancen dürfte das Ausmaß der Vorurteile gegenüber Flüchtlingen in den Wohngebieten unterschiedlich sein. Das ist einerseits ein Kompositionseffekt, andererseits weisen die Gebiete auch ein unterschiedliches soziales Kapital auf: Sie variieren in ihrem wahrgenommenen Vertrauen und der Kohäsion (collective efficacy) (Sampson/Raudenbush/Earls 1997).

\subsection{Hypothesen}

Es geht uns zuerst darum zu beschreiben, wie die Einstellungen der Bewohner der Wohngebiete zu Flüchtlingen allgemein und zu der Flüchtlingsunterkunft speziell sind.

\section{H1: Einstellungen zu den Flüchtlingen}

1.1. Wir nehmen an, dass ein großer Teil der deutschen Bevölkerung eine positive Einstellung zu Flüchtlingen - zumindest zu denen aus Kriegsgebieten und jenen, die politisch verfolgt werden - aufweist. Dafür sprechen Umfragedaten ${ }^{1}$, die breite Unterstützung der Flüchtlinge durch Spenden und das erhebliche Ausmaß freiwilliger (ehrenamtlicher) Arbeit, das zu Spitzenzeiten bei fast $10 \%$ der Gesamtbevölkerung lag (Jacobsen/ Eisnecker/Schupp 2017; Ahrens 2017: 16). 1.2. Zugleich gehen wir in Anlehnung an Allport (1954) davon aus, dass die Einstellung zu den Flüchtlingen nach soziodemographischen Variablen variiert: negativ mit dem Alter, und positiv mit dem Einkommen und dem Bildungsniveau. Mit einem höheren sozialen Status sollten daher positivere Einstellungen einhergehen. 1.3. Weiterhin lässt sich aus der Hypothese 1.2. ableiten, dass auch die soziale Zusammensetzung der Wohngebiete einen Einfluss auf das Akzeptanzniveau haben sollte. So ist für statushohe Gebiete wie HamburgHarvestehude oder Köln-Rondorf ein kollektiv positiveres Einstellungsklima zu erwarten. In statusniedrigeren Wohngebieten wie Mülheim-Mitte oder Köln-Ostheim hingegen sollten die Einstellungen ablehnender sein.

1 Im ALLBUS (Allgemeine Bevölkerungsumfrage der Sozialwissenschaften) 2016 befürworten rund $40 \%$ der Befragten einen uneingeschränkten Zuzug von Kriegsflüchtlingen (eigene Berechnung mit Daten des ALLBUS 2016).

\section{H2: Einstellung zu den Flüchtlingsunterkünften}

2.1. Die Bewohner hatten sich ein Wohngebiet nach ihren Präferenzen und Ressourcen ausgesucht - Flüchtlinge waren kein Bestandteil der Entscheidung. Nun aber wurde in ihrem Wohngebiet eine Unterkunft errichtet, die sie akzeptieren müssen. Wir nehmen an, dass der unerwartete Eingriff in das Wohngebiet, dort eine Unterkunft für Flüchtlinge zu schaffen, von der Mehrzahl der Bewohner abgelehnt wird und zu einer negativen Einstellung führt. 2.2. Das führt uns zu einer weiteren Annahme, dass die Einstellungen konfligieren: Flüchtlinge werden zwar willkommen geheißen, im eigenen Wohngebiet wird eine Unterbringung jedoch abgelehnt (not in my backyard). 2.3. Gleichzeitig nehmen wir an, dass Individuen, die generell ein höheres Angstniveau gegenüber Flüchtlingen aufweisen, auch einen geringeren Anteil an Flüchtlingen in ihrem Umfeld akzeptieren.

\section{H3: Kontakte}

Darüber hinaus gehen wir davon aus, dass direkte und indirekte Kontakte zu Flüchtlingen mit geringeren Vorurteilen der Beteiligten verbunden sind.

\section{H4: Soziales Kapital}

Das Konzept collective efficacy wurde bislang nicht auf die Akzeptanz von Minoritäten im Wohngebiet angewendet. Nachgewiesen wurde, dass hohe collective efficacy in einem Wohngebiet mit niedriger Kriminalität und niedriger Verwahrlosung verbunden ist; sie ist niedrig in Gebieten mit einem hohen Anteil von Minoritäten (Sampson 2012: 168 ff.). Wir vermuten daher, dass mit steigendem sozialen Kapital (collective efficacy), die Akzeptanz der Flüchtlinge geringer ist.

\section{H5: Effekte von Stadt und Wohngebiet}

Eine weitere Überlegung bezieht sich auf den Zusammenhang zwischen der Größe der Stadt und der Akzeptanz der Flüchtlingsunterkunft in den einzelnen Wohngebieten. Wir prüfen diese Annahme indem wir testen, ob die Unterschiede zwischen Wohngebieten der gleichen Stadt geringer sind als die Unterschiede zwischen gleichartigen Wohngebieten zweier verschiedener Städte. 


\subsection{Operationalisierungen}

Um soziale Erwünschtheit zu vermeiden, haben wir verschiedene Zugriffe auf die Einstellungsmessung gewählt: offene Fragen und für jedes Konzept mehrere Indikatoren. Zuerst haben wir die Einstellungen zum Zuzug von Flüchtlingen durch folgende Fragen (übernommen aus dem ALLBUS 2016) erhoben: „Im Folgenden geht es um den Zuzug verschiedener Personengruppen nach Deutschland. Wie ist Ihre Einstellung dazu? 1. Wie ist es mit Flüchtlingen aus Ländern, in denen Krieg herrscht? 2. Und mit Flüchtlingen, die in ihrer Heimat politisch verfolgt werden? 3 . Und mit Flüchtlingen, die wegen der wirtschaftlichen Not in ihren Heimatländern nach Deutschland kommen?" Die Antwortvorgaben lauteten: „Der Zuzug soll UNEINGESCHRÄNKT möglich sein; Der Zuzug soll BEGRENZT werden; Der Zuzug soll völlig UNTERBUNDEN werden; Weiß nicht".

Die Einstellungen zu Flüchtlingen wurden durch zwei offene Fragen erhoben: „Was haben Sie über die Flüchtlinge gedacht, bevor die Flüchtlingsdiskussion aufkam?" (nachfolgend als "ursprüngliche Einstellung" bezeichnet) und "Was denken Sie heute?" (nachfolgend als „aktuelle Einstellung“ bezeichnet). Die Einstellungen zur jeweiligen Flüchtlingsunterkunft wurden über drei offene Fragen gemessen: „In [Ort der Unterkunft] ist ein Flüchtlingsheim eingerichtet worden. Dazu gibt es verschiedene Meinungen. Was denken Sie darüber?", "Welche Nachteile hat das Heim in diesem Wohngebiet?" und "Welche Vorteile hat es?".

Die Befürchtungen oder Ängste der Bewohner gegenüber Flüchtlingen haben wir mit einer Skala gemessen, die von infratest dimap seit 2015 mehrfach in ihren ARD-Monatstrends verwendet wurde (vgl. Infratest-dimap 2016). Sie besteht aus elf Punkten, die alle mit „Ich befürchte ..." eingeleitet werden, z. B. „... dass der Einfluss fremder Kulturen in Deutschland zunimmt" (vgl. Tabelle 6). Die Antwortvorgaben sind: „stimme voll zu - stimme eher zu - stimme eher nicht zu - stimme gar nicht zu“. Diese Angst-Skala hat für unsere Daten einen starken ersten Faktor und ist damit eindimensional. Somit ist sie für unsere Analyse gut geeignet. ${ }^{2}$

Ein anderes Verfahren, die Einstellungen zu Minoritäten zu ermitteln, ist, danach zu fragen, welchen Anteil Flüchtlinge man im Wohngebiet akzeptieren würde. Wir haben dazu ein von Farley, Schuman, Bianchi et al. (1978) entwickeltes Verfahren angewendet. Den Befragten wurden 12 Abbildungen (bei Farley/Schuman/Bianchi

2 Verfahren: Kategoriale Hauptkomponentenanalyse, Eigenwert: 5.342, Reliabilität: $\alpha=.894$.
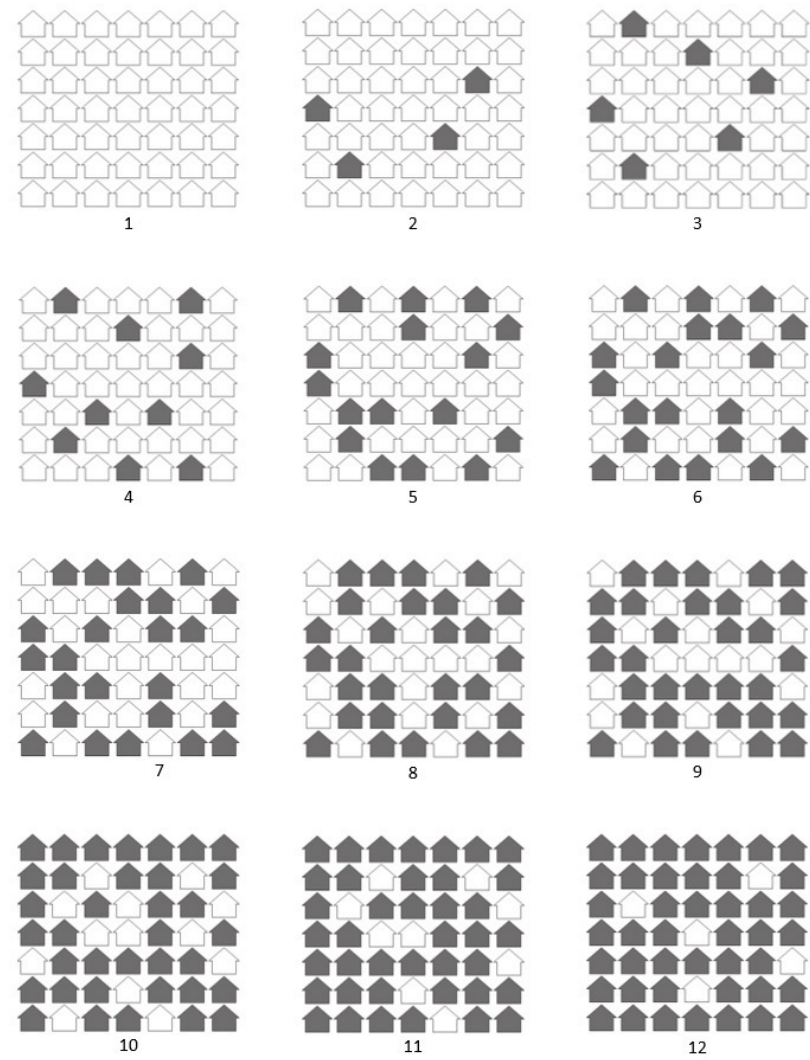

Abbildung 1: Das Farley-Verfahren

et al. waren es 13) mit 7x7 Häusern gezeigt. Von Abbildung zu Abbildung nimmt der Anteil eingefärbter Häuser (bzw. Bewohner) zu (vgl. Abbildung 1). Wir stellten dazu folgende Frage: „Wir haben hier auf der Liste Wohngebiete dargestellt, die sich im Anteil an Flüchtlingen als Nachbarn unterscheiden. Die hellen Felder stellen dabei deutsche Personen dar, die grauen Felder stehen für Flüchtlinge. Nennen Sie bitte die Nummern aller Wohngebiete, in denen Sie gerne wohnen würden." Dieses Verfahren ist in Deutschland im ALLBUS 2006 und 2016 (GESIS 2011; GESIS 2017) angewendet worden.

Zu den Kontakten stellten wir die Fragen, ob man persönlich Kontakt zu Flüchtlingen hat oder ob man indirekte Kontakte zu Flüchtlingen über Familienangehörige oder Freunde hat (beides mit Ja-Nein codiert). ${ }^{3}$ Die Beschreibung der Gebiete enthält die Unterkunft, soziodemographische Merkmale der Bewohner und ökonomische Indikatoren. Zusätzlich messen wir die Kohäsion und das Vertrauen in die Nachbarschaft mit-

3 Über weitere Nachfragen zur Art des Kontaktes konnten ganz oberflächliche Begegnungen identifiziert werden. Diese wurden nicht als Kontakt gezählt. 
hilfe der Skala der kollektiven Effektivität (collective efficacy) von Sampson (Sampson/Groves 1989; Sampson/ Raudenbush/Earls 1997; Sampson 2012). Wir folgen den Autoren und bezeichnen dies als „soziales Kapital“. Die Skala hat fünf Punkte: „Die Leute hier helfen sich gegenseitig.“, „Hier kennen sich die Leute gut.." „Man kann den Leuten in der Nachbarschaft vertrauen.“, „Die Leute hier kommen gut miteinander aus.“, „Die Leute hier haben Respekt vor Gesetz und Ordnung.“. Die Antwortvorgaben auf die Frage: „Wenn Sie an Ihre Nachbarschaft denken: Inwieweit treffen die folgenden Aussagen Ihrer Meinung nach zu?" lauten: „trifft voll zu - trifft zu - trifft eher nicht zu - trifft nicht zu“. Diese übersetzte Skala hat sich auch in mehreren deutschen Studien bewährt (Oberwittler 2004; Blasius/Friedrichs 2007; Blasius/ Friedrichs/Klöckner 2008). Die Variable geht über einen additiven Index der Zustimmungen in die Analyse ein.

\section{Untersuchungsgebiete, Methoden und Stichproben}

\subsection{Beschreibung der Untersuchungsgebiete}

In jeder der drei Städte, Hamburg, Köln und Mülheim an der Ruhr, wurde mit Experten der Stadtplanung über die Auswahl von zwei unterschiedlichen Wohngebieten gesprochen. Die Gebiete sollten sich nach der Sozialstruktur und nach der Art der Unterkunft unterscheiden (vgl. Tabellen 1, 2 und 3).

\subsubsection{Hamburg}

\subsubsection{Harvestehude}

Die Flüchtlingsunterkunft Sophienterrasse liegt im Stadtteil Harvestehude. In dem ehemaligen Kreiswehrersatzamt sollten ursprünglich 220 Flüchtlinge untergebracht werden. Dagegen haben Anwohner geklagt, unter anderem, weil sie eine solche Nutzung in einem reinen Wohngebiet nicht für zulässig hielten. Durch eine außergerichtliche Einigung auf die Begrenzung der Bewohnerzahl auf 190 Flüchtlinge konnte die Unterkunft ab Mitte Februar 2016 bezogen werden. Es wurden fast ausschließlich Familien in den abgetrennten Wohneinheiten untergebracht. Eine Selbstversorgung ist möglich, wobei die sanitären Anlagen zum Teil von zwei Familien geteilt werden. Harvestehude ist ein Wohngebiet der oberen Mittelschicht und Oberschicht. Es ist sehr homogen.
Deutlich wird dies unter anderem am Lohn- und Einkommenssteuersatz je Steuerpflichtigem. Gleichzeitig liegt der Anteil an Hartz-IV-Empfängern mit 3,6 \% deutlich unterhalb der gesamtstädtischen Quote von 9,9\%. Darüber hinaus geht aus unserer Stichprobe hervor, dass auch das Bildungsniveau weit über dem Durchschnitt liegt.

\subsubsection{Bergedorf}

Die Flüchtlingsunterkunft Brookkehre liegt am südlichen Rand eines Wohngebiets, fast in einem Gewerbegebiet. Die Unterkunft für 380 bis maximal 440 Personen wurde relativ schnell errichtet und im Juni 2015 bezogen. Sie besteht aus dreistöckigen, grün gestrichenen Modulhäusern, die in jeweils mehrere Dreizimmerwohnungen mit eigener Küche und Bad unterteilt sind. Bei der Belegung wurden bevorzugt Familien ausgewählt. Neben dieser bestehen über den Stadtteil Bergedorf verteilt sechs weitere Flüchtlingsunterkünfte. Das Wohngebiet weist die klassischen Merkmale eines Mittelschicht-Gebiets auf, was sich an den mittleren Einkommen und einem überschaubaren Anteil an Empfängern von Sozialleistungen und der Arbeitslosenquote zeigt. Im Gegensatz zu Harvestehude gab es hier, wie auch in allen anderen Gebieten, zwar vereinzelt Anwohnerproteste gegen die Errichtung der Unterkunft, jedoch keine gerichtlichen Verfahren.

\subsubsection{Köln}

\subsubsection{Ostheim}

Im Januar 2016 wurden am Hardtgenbuscher Kirchweg fünf neu errichtete Leichtbauhallen mit bis zu 400 Flüchtlingen bezogen. In den Hallen sind jeweils 80 Personen untergebracht. Zunächst gab es keine Abtrennung zwischen den Betten, nach etwa neun Monaten wurden die Hallen dann jedoch mit nach oben offenen Kojen ausgestattet, um ein Mindestmaß an Privatsphäre zu gewährleisten. Eine selbstständige Verpflegung der Bewohner ist nicht möglich (Vollverpflegung in der Halle). Anfang 2018 konnten die Hallen geräumt und die Bewohner auf andere Standorte verlegt werden. Ostheim zeigt Merkmale eines unteren Mittel- bis Unterschicht-Wohngebiets, in dem es zwei Armutsgebiete gibt. Auffällig sind die hohe Ausländerquote, der vergleichsweise geringe Anteil an Vielverdienern und hohe Anteil an Geringverdienern, die Arbeitslosenquote sowie der Anteil an SGBII-Empfängern. 
Tabelle 1: Ausgewählte Merkmale der Sozialstruktur von Harvestehude und Bergedorf in Hamburg (2016)

\begin{tabular}{|c|c|c|c|c|c|}
\hline \multirow[t]{2}{*}{ Merkmal } & \multicolumn{2}{|c|}{ Harvestehude } & \multicolumn{2}{|l|}{ Bergedorf } & \multirow[t]{2}{*}{ Hamburg } \\
\hline & Stichprobe & Statistik $^{\mathrm{a}}$ & Stichprobe & Statistik $^{\mathrm{a}}$ & \\
\hline Einwohner & - & 17.822 & - & 34.404 & 1.833 .93 \\
\hline Ausländer & - & $12,8 \%$ & - & $14,0 \%$ & $15,9 \%$ \\
\hline Abitur & $81,9 \%$ & - & $62,8 \%$ & - & - \\
\hline Hochschulstudium & $63,5 \%$ & - & $38,4 \%$ & - & - \\
\hline Einkommen $>5.000 €^{\mathrm{b}}$ & $41,2 \%$ & - & $13,2 \%$ & - & - \\
\hline Einkommen $<1.500 €^{b}$ & $11,5 \%$ & - & $17,9 \%$ & - & - \\
\hline Steuer je Steuerpflichtigem (€) & - & 111.088 & - & 32.742 & 35.567 \\
\hline Wohnungseigentümer & $36,2 \%$ & - & $34,7 \%$ & - & - \\
\hline Sozialmieter & $1,9 \%$ & - & $5,9 \%$ & - & - \\
\hline Eigentumswohnung (€/qm) & - & 8.101 & - & 2.758 & 3.658 \\
\hline Wohnungsgröße (qm) & - & 96,8 & - & 79,5 & 83,6 \\
\hline Arbeitslosenquote & $1,9 \%$ & $3,5 \%$ & $4,7 \%$ & $5,0 \%$ & $5,6 \%$ \\
\hline SGB-II-Empfänger & $0,8 \%$ & $3,6 \%$ & $4,7 \%$ & $9,6 \%$ & $9,9 \%$ \\
\hline
\end{tabular}

a Daten des Statistikamts Nord 2016, ${ }^{\mathrm{b}}$ Haushaltsnetto-Einkommen

\subsubsection{Rondorf}

In Rondorf wurden im Weißdornweg 80 Plätze in abgeschlossenen Wohneinheiten für Familien sowie Wohngemeinschaften geschaffen. Jede Wohneinheit verfügt über ein eigenes Bad und eine Wohnküche und ermöglicht somit Privatsphäre und eine eigenständige Versorgung. Die zweigeschossige Anlage ist verputzt und umzäunt und hat für die jungen Bewohner einen kleinen Spielplatz auf dem Gelände. Die Sozialstruktur Rondorfs ähnelt der von Hamburg-Bergedorf. Es handelt sich hier um ein Gebiet der Mittelschicht mit einem dörflichen Charakter und einer auffällig hohen Wohnungseigentümerquote.

\subsubsection{Mülheim an der Ruhr}

\subsubsection{Mitte}

Mülheim sieht in seinem Konzept öffentlich-rechtlicher Unterbringung vor, Flüchtlinge dezentral in Wohnungen unterzubringen. ${ }^{4}$ Einige Wohnungen befinden sich in Mülheim-Mitte, unter anderem entlang der FriedrichEbert-Straße. Den Anwohnern ist nicht bekannt, in welchen Straßenzügen oder Wohnungen Flüchtlinge

4 Die Kapazitäten an vom Sozialamt angemieteten Wohnungen waren für die Zugangszahlen der letzten Jahre nicht ausgelegt, entsprechend musste die Stadt ab Herbst 2014 zusätzliche zentrale Unterbringungen bereitstellen (Mühlenfeld 2015: 128), ab September 2015 wurden hierzu zeitweise auch Turnhallen genutzt. leben. Die Sozialstruktur von Mülheim-Mitte ähnelt der von Köln-Ostheim. Es handelt sich um ein Gebiet der unteren Mittelschicht und Mittelschicht.

\subsubsection{Saarn}

Die untersuchte Unterkunft in der Mintarder Straße befindet sich auf dem Kirmesplatz am Rande eines Wohngebiets mit dörflichem Charakter. Die Unterkunft für 400 bis maximal 540 Personen wurde ab November 2015 sukzessive bezogen und im Juni 2016 fertig gestellt. Die Erstaufnahmeeinrichtung besteht aus einstöckigen Holzbauten, in denen sich jeweils vier Personen ein Zimmer mit 16 qm teilen. Die Bewohner werden wie in Köln-Ostheim voll verpflegt. ${ }^{5}$ Sanitäranlagen befinden sich zentral auf dem Gelände. Das Befragungsgebiet ähnelt Köln-Rondorf und Hamburg-Bergedorf.

\subsection{Stichprobe und Methoden}

Um für die Befragung der Anwohner zu einer repräsentativen Stichprobe zu gelangen, haben wir in jeder Stadt für jedes der zwei Gebiete eine Wahrscheinlichkeitsstichprobe aus der Datei des jeweiligen Einwohnermeldeamtes für alle Straßen im Umkreis von etwa 2,5 Kilometer um die Unterkünfte ziehen lassen. Der

5 Inzwischen wurden Kochnischen eingerichtet und die Unterkunft auf Selbstverpflegung umgestellt. 
Tabelle 2: Ausgewählte Merkmale der Sozialstruktur von Ostheim und Rondorf in Köln (2016)

\begin{tabular}{|c|c|c|c|c|c|}
\hline \multirow[t]{2}{*}{ Merkmal } & \multicolumn{2}{|l|}{ Ostheim } & \multicolumn{2}{|l|}{ Rondorf } & \multirow[t]{2}{*}{ Köln } \\
\hline & Stichprobe & Statistik $^{a}$ & Stichprobe & Statistik $^{a}$ & \\
\hline Einwohner & - & 13.524 & - & 9.641 & 1.081 .701 \\
\hline Ausländer & - & $32,5 \%$ & - & $14,0 \%$ & $19,3 \%$ \\
\hline Abitur & $50,2 \%$ & - & $57,7 \%$ & - & - \\
\hline Hochschulstudium & $25,3 \%$ & - & $32,6 \%$ & - & - \\
\hline Einkommen $>5.000 €^{b}$ & $10,0 \%$ & - & $31,2 \%$ & - & - \\
\hline Einkommen $<1.500 €^{\mathrm{b}}$ & $28,8 \%$ & - & $12,1 \%$ & - & - \\
\hline Steuer je Steuerpflichtigem (€) & - & - & - & - & - \\
\hline Wohnungseigentümer & $51,7 \%$ & - & $68,4 \%$ & - & - \\
\hline Sozialmieter & $12,6 \%$ & $27,1 \%$ & $1,7 \%$ & $6,1 \%$ & $6,8 \%$ \\
\hline Eigentumswohnung (€/qm) & - & 1.652 & - & 2.408 & 2.928 \\
\hline Wohnungsgröße (qm) & - & 75,0 & - & 92,9 & 72,8 \\
\hline Arbeitslosenquote & $7,2 \%$ & $15,3 \%$ & $1,7 \%$ & $4,4 \%$ & $8,1 \%$ \\
\hline SGB-II-Empfänger & $7,8 \%$ & $28,3 \%$ & $3,5 \%$ & $6,1 \%$ & $13,1 \%$ \\
\hline
\end{tabular}

a Daten der Stadt Köln 2016, ${ }^{b}$ Haushaltsnetto-Einkommen

Tabelle 3: Ausgewählte Merkmale der Sozialstruktur von Mitte und Saarn' in Mülheim an der Ruhr (2016)

\begin{tabular}{|c|c|c|c|c|c|}
\hline \multirow[t]{2}{*}{ Merkmal } & \multicolumn{2}{|l|}{ Mitte } & \multicolumn{2}{|l|}{ Saarn } & \multirow{2}{*}{$\begin{array}{l}\text { Mülheim an der } \\
\text { Ruhr }\end{array}$} \\
\hline & Stichprobe & Statistik $^{a}$ & Stichprobe & Statistik $^{a}$ & \\
\hline Einwohner & - & 15.258 & - & 14.148 & 172.593 \\
\hline Ausländer & - & $31,0 \%$ & - & $9,0 \%$ & $14,4 \%$ \\
\hline Abitur & $58,2 \%$ & - & $54,7 \%$ & - & - \\
\hline Hochschulstudium & $28,4 \%$ & - & $32,3 \%$ & - & - \\
\hline Einkommen $>5.000 €^{\mathrm{c}}$ & $8,3 \%$ & - & $13,4 \%$ & - & - \\
\hline Einkommen $<1.500 €^{c}$ & $31,7 \%$ & - & $22,4 \%$ & - & - \\
\hline Steuer je Steuerpflichtigem $(€)$ & - & - & - & - & - \\
\hline Wohnungseigentümer & $19,7 \%$ & - & $43,2 \%$ & - & - \\
\hline Sozialmieter & $10,9 \%$ & - & $1,9 \%$ & - & - \\
\hline Eigentumswohnung (€/qm) & - & - & - & - & - \\
\hline Wohnungsgröße (qm) & - & - & - & - & 79,9 \\
\hline Arbeitslosenquote & $6,7 \%$ & $10,3 \%$ & $1,1 \%$ & $3,8 \%$ & $6,1 \%$ \\
\hline SGB-II-Empfänger & $14,9 \%$ & $30,3 \%$ & $0,8 \%$ & $7,8 \%$ & $15,9 \%$ \\
\hline
\end{tabular}

a Daten der Stadt Mülheim an der Ruhr 2016; ${ }^{\mathrm{b}}$ Mitte = Stadtteile: 11, 12, 24; Saarn = Stadtteil $71 ;{ }^{\mathrm{c}}$ Haushaltsnetto-Einkommen

standardisierte Fragebogen wurde in einer Studie von Michaelis (2016) in einem Kölner Wohngebiet verwendet, überarbeitet und dann einem Pretest unterzogen. Die Erhebung erfolgte face-to-face von März bis Juli 2016 in Hamburg-Harvestehude, November 2016 bis Juni 2017 in Hamburg-Bergedorf, November 2016 bis Juni 2017 in Köln und November 2016 bis Dezember 2017 in Mülheim an der Ruhr. Die Befragung wurde von Studierenden durchgeführt, die wir ausführlich geschult hatten. Die ausgefüllten Fragebögen wurden auf die Vollständigkeit der Antworten und das Geschlecht der befragten Person hin kontrolliert. Für die Codierung der offenen Fragen erstellten wir einen dreistufigen CodierLeitfaden (Mayring 2010: 92), bestehend aus (1) der genauen Definition der Kategorien, (2) der Identifikation von Ankerbeispielen, die beispielhaft für eine Kategorie konkrete Zitate anführen sowie (3) der Festlegung von Codier-Regeln, die eine eindeutige Zuordnung bei Abgrenzungsproblemen ermöglichen sollen.

Gemessen an anderen sozialwissenschaftlichen Studien, z. B. dem ALLBUS (Ausschöpfung 2016: $34,6 \%$ ), ist die Ausschöpfungsquote von durchschnitt- 
lich $38,6 \%$ durchaus akzeptabel. Aufgrund des hohen Anteils von Einpersonenhaushalten in Hamburg-Harvestehude hatten wir beträchtliche Schwierigkeiten, die Befragten zu erreichen. In Hamburg-Bergedorf und Mülheim-Mitte hingegen hatten wir erheblich mehr Ausfälle aufgrund von Verweigerungen.

Wir haben geprüft, ob sich Personen, die ein Interview verweigerten, von denjenigen unterscheiden, die sich haben interviewen lassen. Mit den uns verfügbaren Mitteln - einem Vergleich nach Geschlecht und Alter hat es keine selektiven Ausfälle nach den Einstellungen der Befragten gegeben. Dennoch können wir nicht ausschließen, dass eher Personen, die der Flüchtlingspolitik gegenüber ablehnend eingestellt sind, das Interview verweigert haben.

\section{Ergebnisse}

Die Ergebnisse stellen wir in der Reihenfolge der Hypothesen dar. Somit behandeln wir zuerst die Ergebnisse zur Einstellung zu Flüchtlingen generell, dann die Einstellung zur Unterkunft, anschließend die geäußerten Befürchtungen und abschließend die Antworten auf die Fragen nach bestehenden Kontakten. In Kapitel 4.6 führen wir die Ergebnisse mithilfe einer Korrespondenzanalyse zusammen.

\subsection{Einstellungen zu den Flüchtlingen}

Im Jahr 2015 kamen fast 900.000 Flüchtlinge nach Deutschland. Der Aussage, „Es macht mir Angst, dass viele Flüchtlinge zu uns kommen.", stimmten im Februar 2016 bundesweit 49 \% der Befragten „,eher zu“ (Infratestdimap 2016: 1). In unseren Wohngebieten waren es in Hamburg-Harvestehude 18,3\%, in Hamburg-Bergedorf 25,0 \%, in Köln-Ostheim 33,7 \%, in Köln-Rondorf 25,1 \%, in Mülheim-Mitte 33,5 \% und in Mülheim-Saarn 27,1 \%; es sind damit erheblich weniger als in der zitierten Studie.

Um herauszufinden, was die Befragten generell über Flüchtlinge denken, haben wir offen gefragt, was sie vor der Flüchtlingsdiskussion über Flüchtlinge gedacht haben und was sie heute meinen. Die Antworten auf diese Fragen waren sehr unterschiedlich und zahlreich. Wir haben bei der Codierung bis zu drei Antworten berücksichtigt, daher der hohe Anteil von „Sonstiges“. Bei den ursprünglichen Einstellungen sagt über die Hälfte der Befragten, sie verspürte Solidarität oder hätte Mitgefühl. Dazu kommen noch hohe Anteile, die nur "sehe ich positiv“ antworten. Am wenigsten Solidari- tät äußern die Befragten in Köln-Rondorf und MülheimMitte, am meisten diejenigen in Hamburg-Harvestehude. In Köln-Rondorf könnte das darauf zurückzuführen sein, dass das Wohngebiet einen dörflichen, abgeschlossenen Charakter hat. Im Falle von Mülheim-Mitte gibt es keine Unterkunft, die Flüchtlinge sind über Wohnungen in einem Teil der Innenstadt verstreut. Hier wohnen auch viele Migranten, von denen ein Teil den Flüchtlingen skeptisch gegenübersteht. Aufschlussreich ist, dass sich trotz der Berichte und Debatten über Flüchtlinge seit 2012 ein Drittel keine Gedanken über Flüchtlinge gemacht hat und sich als „uninformiert" bezeichnet.

Im Folgenden zitieren wir einige Antworten, die einen Eindruck von der Spannweite der Ansichten vermitteln: „Ich finde, dass das ganz arme Menschen sind und wir bereit sind zu helfen. Die ganze Diskussion ist überflüssig und peinlich für Deutschland“ (H-Bgd. 2644). „Was ich nicht gut finde, ist, dass jetzt mehr Gewalt hier herrscht. Sie werden besser behandelt als wir: Harz IV, Busticket [...] Sie bekommen einen Freifahrtschein für alles. Das ist uns allen gegenüber nicht fair. Wut kommt raus. Sie sollten alle wieder nach Hause geschickt werden" (H-Harv. 715).

Die aktuellen Einstellungen zu den Flüchtlingen zeigen etwas weniger Solidarität und mehr zwiegespaltene Personen. Ein nicht geringer Anteil meint, es kämen zu viele Flüchtlinge (insbesondere in Köln-Ostheim) oder ist für Zuzugskontrollen (insbesondere in MülheimSaarn) oder für Hilfen im Herkunftsland statt in Deutschland. Rund $11 \%$ nennen ein Versagen der Politik, nicht rechtzeitig reagiert zu haben, sowohl auf europäischer Ebene, aber auch was die Registrierung der Flüchtlinge angeht. Andere führen Bedingungen auf: Die Flüchtlinge sind willkommen, wenn sie sich anpassen. Auch hier einige Beispiele der Antworten: „Ich habe durch den Flüchtlingsstrom sehr viele Ängste um die Zukunft: dass die Kriminalität steigt, dass das Bildungsniveau in den Schulen sinkt und die Rechten an die Macht kommen und somit die politische Situation im Land beeinflussen. Schlimm ist auch, dass es überhaupt keine Kontrolle gibt, wer reinkommen darf und wer nicht. Die Terrorgefahr steigt gewaltig“ (H-Bgd. 2725). „Ich wäre auch gern Flüchtling!!! Luxuriöse, neue, saubere Unterkunft, Nahrung unter Berücksichtigung eventueller Vorlieben/ Glaubensbekennung etc., etc., etc." (K-Osth. 7821). „Uns geht es hier gut, habe durch die Flüchtlinge nicht weniger Butter auf dem Brot“" (K-Rond. 8918).

Die Einstellung zu Flüchtlingen ist wie erwartet positiv. Die positiven Urteile sind am höchsten in Hamburg-Harvestehude und Köln-Rondorf - also den beiden statushöchsten Gebieten - und am niedrigsten in Mül- 
heim-Mitte und Mülheim-Saarn. Das entspricht unserer Hypothese 1.3.: Je höher der soziale Status eines Wohngebiets, desto eher werden Flüchtlinge und Unterkünfte akzeptiert. Die Einstellungen weisen keinen signifikanten Zusammenhang mit dem Geschlecht und dem Alter der Befragten auf (H1.2.). Hingegen nehmen mit steigender Schulbildung und steigendem Einkommen die positiven Antworten und die Solidarität signifikant zu, der Anteil der ,Uninformierten' sinkt signifikant.

\subsection{Akzeptanz von Flüchtlingen nach Zuzugsgründen}

Die Einstellungen zu Flüchtlingen sind stark abhängig davon, aus welchen Gründen die Flüchtlinge kommen. Krieg und politische Verfolgung werden sehr viel stärker akzeptiert als wirtschaftliche Gründe. Aus der Sicht der Flüchtlinge sind gewaltsame Konflikte und Krieg bei $70 \%$ auch der wichtigste Grund, das Herkunftsland zu verlassen, gefolgt von $44 \%$, die „Verfolgung“ als Grund angeben (Brücker/Rother/Schupp 2016: 16). Wir haben daher gefragt, ob man Flüchtlinge eher aus wirtschaftlichen, politischen oder Kriegsgründen akzeptieren würde (vgl. Tabelle 4 und Kapitel 2.4). Die Ergebnisse hierzu sind über die Gebiete erstaunlich einheitlich. Es zeigt sich, dass die Akzeptanz von Kriegs- und politischen Flüchtlingen über alle Gebiete sehr hoch ist. Dagegen wünschen 59 \% der Befragten, den Zuzug von Flüchtlingen, die aus wirtschaftlichen Gründen kommen, zu begrenzen; weitere $30 \%$ lehnen deren Zuzug völlig ab. Die Ablehnung wirtschaftlicher Flüchtlinge ist in den beiden Gebieten mit dem höchsten sozialen Status, Hamburg-Harvestehude und Köln-Rondorf, am höchsten, in Köln-Ostheim, als statusniedrigem Gebiet ist die Ablehnung dieser Gruppe hingegen am niedrigsten.

Aus der Kombination dieser drei Fragen und Antworten haben wir acht Typen gebildet. Die wichtigsten sind in Tabelle 5 aufgeführt und nach zentralen unabhängigen Variablen differenziert. Der durchgängig häufigste Typ ist T2: Flüchtlinge, die aus politischen Gründen oder aufgrund von (Bürger-)Krieg kommen, werden völlig akzeptiert, solche aus wirtschaftlichen Gründen hingegen nicht.

Jüngere Befragte sind toleranter als ältere, die auch häufiger die restriktivsten Antworten wählen. Bei der Schulbildung gibt es ebenfalls ein klares Gefälle. Die Verteilung nach dem Einkommen ist eher kurvilinear: Die Werte für Typ 6 steigen, fallen dann aber mit höheren Einkommen wieder. Bezieher von Sozialhilfe (Arbeitslosengeld, Hartz IV) haben signifikant positivere Einstellungen zum Zuzug von Flüchtlingen als Personen, die keine Sozialhilfe erhalten. Das ist ein unerwartetes Ergebnis, denn man hätte davon ausgehen können, Bezieher von Sozialhilfe sähen in den Flüchtlingen eine Konkurrenz um knappe Sozialbudgets. Der Zuzug von Flüchtlingen weist einen engen linearen Zusammenhang mit der Toleranz (vgl. Kapitel 2.4 Farley-Verfahren) auf: Wer mehr Flüchtlinge im Wohngebiet akzeptiert, lässt auch Flüchtlinge aller Fluchtgründe eher zu. Alle bislang aufgeführten Zusammenhänge sind signifikant auf $p<.001$.

Für die sechs Wohngebiete zeichnen sich keine signifikanten Unterschiede ab. Die (reicheren) Haushalte in Hamburg-Harvestehude lehnen die Wirtschaftsflüchtlinge stärker ab als die Bewohner anderer Gebiete. Auffällig sind die relativ hohen Anteile für Typ 6 in HamburgBergedorf, Köln-Rondorf und Mülheim-Saarn. Hingegen sind die Bewohner von Mülheim-Mitte, dem einzigen Untersuchungsgebiet mit dezentraler Unterbringung, relativ am tolerantesten.

\subsection{Einstellungen zu den Flüchtlingsunterkünften}

Mit drei offenen Fragen haben wir erhoben, wie die Anwohner über die jeweilige Unterkunft denken, welche Vor- und welche Nachteile die Unterkunft ihrer Ansicht nach hat. Insgesamt sind die Einstellungen sehr positiv, insbesondere in Hamburg-Harvestehude und KölnRondorf. Weniger als $10 \%$ äußern sich negativ. Sehr unterschiedlich sind die Anteile derer, die sagen, sie seien uninformiert. Das geht auf die Lage der Unterkünfte im jeweiligen Wohngebiet zurück: Liegt die Unterkunft peripher, wird sie weniger wahrgenommen, so z. B. in Hamburg-Bergedorf. In Mülheim-Mitte gibt es keine sichtbare Unterkunft. Hinter der Antwortkategorie "Gegend unpassend“ verbergen sich in Hamburg-Harvestehude Befürchtungen der Befragten, der Kontrast von Reichtum des Gebiets und Status der Flüchtlinge sei zu krass. Erstaunlich ist, dass nach Protesten auf Bürgerversammlungen, die es in allen Untersuchungsgebieten gab, nun so wenige Befragte sagen, man habe kein Mitspracherecht gehabt. Nachfolgend einige Antwortbeispiele: „Der ungünstigste Platz, den man finden konnte. Stand vorher für Anwohner zur Verfügung. Andere Plätze wären besser geeignet. Zu abgeschottet" (M-Saarn 6238). „Wo sollen die sein? Ich habe nichts mitgekriegt" (M-Mitte 4560). „Am Anfang, als das rauskam, dachten wir schon, dass das verdammt nah ist! Aber wir haben keinerlei Probleme damit. Wir sind ohnehin ,multikulti“ hier und es klappt ganz gut" (K-Osth. 7274). 
Tabelle 4: Einstellungen zum Zuzug von Flüchtlingen, nach Wohngebiet, in Prozent

\begin{tabular}{|c|c|c|c|c|c|c|c|}
\hline \multirow{2}{*}{$\begin{array}{l}\text { Zuzug } \ldots . \\
\text { sollte ..... }\end{array}$} & \multicolumn{2}{|l|}{ Hamburg } & \multicolumn{2}{|l|}{ Köln } & \multicolumn{2}{|c|}{ Mülheim an der Ruhr } & \multirow{2}{*}{$\begin{array}{l}\text { Gesamt } \\
\%\end{array}$} \\
\hline & Harvestehude & Bergedorf & Ostheim & Rondorf & Mitte & Saarn & \\
\hline \multicolumn{8}{|l|}{ aus Kriegsgebieten } \\
\hline $\begin{array}{l}\text { uneingeschränkt } \\
\text { möglich sein }\end{array}$ & 63,0 & 59,3 & 54,2 & 51,6 & 59,9 & 52,7 & 56,5 \\
\hline begrenzt werden & 35,9 & 40,0 & 44,9 & 47,9 & 36,7 & 45,4 & 42,2 \\
\hline unterbunden werden & 1,1 & 0,7 & 1,0 & 0,6 & 3,4 & 1,9 & 1,3 \\
\hline \multicolumn{8}{|l|}{ für politisch Verfolgte } \\
\hline $\begin{array}{l}\text { uneingeschränkt möglich } \\
\text { sein }\end{array}$ & 61,2 & 55,9 & 44,6 & 41,5 & 50,9 & 48,3 & 50,0 \\
\hline begrenzt werden & 36,3 & 42,4 & 48,8 & 55,6 & 41,9 & 47,5 & 45,9 \\
\hline unterbunden werden & 2,6 & 1,7 & 6,8 & 2,9 & 7,3 & 4,2 & 4,1 \\
\hline \multicolumn{8}{|l|}{$\begin{array}{l}\text { aus wirtschaftlichen } \\
\text { Gründen }\end{array}$} \\
\hline $\begin{array}{l}\text { uneingeschränkt möglich } \\
\text { sein }\end{array}$ & 9,0 & 12,4 & 9,9 & 8,0 & 17,2 & 8,8 & 10,7 \\
\hline begrenzt werden & 62,3 & 57,4 & 62,7 & 60,1 & 53,4 & 55,2 & 58,8 \\
\hline unterbunden werden & 28,7 & 30,1 & 27,4 & 31,8 & 29,3 & 36,0 & 30,6 \\
\hline
\end{tabular}

Signifikanzen: Kriegsgebiete: $\mathrm{Chi}^{2}=24,55, \mathrm{p}<.006$; Politisch Verfolgte: $\mathrm{Chi}^{2}=47,82, \mathrm{p}<.001$; Wirtschaftliche Gründe: $\mathrm{Chi}^{2}=21,45, \mathrm{p}<.018$.

In den Befragungen wurden Nachteile und Vorteile der jeweiligen Einrichtung erfasst. Die Hälfte der Befragten nennt keine Nachteile - ein Ergebnis, das viele Stadtplaner beruhigen dürfte. Der Wert ist mit $42 \%$ in Mülheim-Saarn am niedrigsten. In Mülheim-Mitte werden am häufigsten „Konflikte“ genannt, was wir erneut auf die soziale Mischung in dem Gebiet zurückführen. Das Gefühl der Unsicherheit ist in Hamburg-Harvestehude und Köln-Rondorf am höchsten; dies sind auch die statushöchsten Gebiete. Eine spezielle Kategorie sind die Antworten, in denen die Befragten etwas anführen, was die Nachbarn vermuten: mehr Lärm, höhere Unsicherheit und Kriminalität. Wir haben diese Antworten mit anderen Antworten der Befragten verglichen, um herauszufinden, ob eigentlich sie und nicht die Nachbarn solche Bedenken haben, aber das hat sich nicht bestätigt. In Hamburg-Harvestehude werden als Nachteil häufig die „Preise“ genannt; gemeint sind damit die hohen Preise für Lebensmittel, die sich die Flüchtlinge nicht leisten könnten. Das hat ein Befragter (H-Harv. 584) folgendermaßen kommentiert: „Diese Menschen sind tausende von Metern gegangen, die 100 Meter zu Aldi werden sie auch noch schaffen." Schließlich wird auch eine Abwer- tung befürchtet - ein Argument, das in zahlreichen Diskussionen über den Standort einer Flüchtlingsunterkunft angeführt wird, aber angesichts der stetig steigenden Immobilienpreise und der bei einigen Unterkünften bestehenden Befristung der Flüchtlingsunterbringung wenig realistisch erscheint. Nachfolgend weitere Antwortbeispiele: „Es herrscht keine Ruhe, ich habe Angst, vor allem vor männlichen Flüchtlingen“ (M-Mitte 4717). „Entwertung der Grundstücke, genau wie wenn ein Farbiger nebenan einzieht" (K-Rond. 9055). „Ich werde als Ausländerin auch schief angeguckt und für das respektlose Verhalten der Flüchtlinge mit bösen Blicken bestraft. Die Deutschen sehen uns gleich" (M-Saarn 6384).

Auch bei der Frage nach den Vorteilen streut die Antwort "keine" stärker als die Nachteile über die Gebiete. Der Anteil von "keine Vorteile" nimmt in folgender Reihenfolge zu: Hamburg-Harvestehude - Mülheim-Mitte - Köln-Rondorf - Hamburg-Bergedorf - Mülheim-Saarn - Köln-Ostheim. Die Vorteile werden vor allem für das (künftige) Zusammenleben, die Infrastruktur der Gebiete und die Sicherheit der Flüchtlinge gesehen. In HamburgHarvestehude ist ein weiterer genannter Vorteil, dass die Anwohner nun mit der Realität konfrontiert werden und 
Tabelle 5: Zuzug von Flüchtlingen, Antwort-Typenª, nach soziodemographischen Merkmalen, in Zeilen-Prozent

\begin{tabular}{|c|c|c|c|}
\hline Merkmal & $\begin{array}{l}\text { Typ 1: } \\
\text { Alle uneingeschränkt }\end{array}$ & $\begin{array}{l}\text { Typ 2: } \\
\text { zwei von drei uneinge- } \\
\text { schränkt, einer von drei } \\
\text { beschränkt }\end{array}$ & $\begin{array}{l}\text { Typ 6: } \\
\text { zwei von drei beschränkt, } \\
\text { einer von drei völlig } \\
\text { unterbunden }\end{array}$ \\
\hline \multicolumn{4}{|l|}{ Alter } \\
\hline $18-29$ & 12,4 & 32,5 & 7,7 \\
\hline $30-39$ & 15,7 & 28,4 & 9,6 \\
\hline $40-49$ & 7,0 & 30,4 & 12,5 \\
\hline $50-59$ & 6,9 & 28,4 & 16,2 \\
\hline $60-69$ & 5,7 & 29,0 & 19,8 \\
\hline $70+$ & 7,4 & 25,2 & 21,0 \\
\hline \multicolumn{4}{|l|}{ Schulbildung ${ }^{b}$} \\
\hline Volks-/Hauptschule & 7,0 & 19,2 & 25,8 \\
\hline Mittlere Reife & 5,5 & 21,8 & 21,2 \\
\hline Abitur & 11,7 & 26,9 & 12,5 \\
\hline Fach-/Hochschule & 9,4 & 37,5 & 9,4 \\
\hline \multicolumn{4}{|l|}{ Einkommen $(€)$} \\
\hline-999 & 10,7 & 27,9 & 13,9 \\
\hline $1000-1499$ & 11,2 & 28,5 & 16,9 \\
\hline 1500-1999 & 12,1 & 26,6 & 17,6 \\
\hline 2000-2499 & 8,4 & 28,1 & 13,2 \\
\hline $2500-3499$ & 6,4 & 33,2 & 11,5 \\
\hline $3500+$ & 7,8 & 38,8 & 11,0 \\
\hline \multicolumn{4}{|l|}{ Sozialhilfe ${ }^{c}$} \\
\hline $\mathrm{Ja}$ & 18,5 & 29,6 & 3,7 \\
\hline Nein & 8,5 & 28,5 & 15,5 \\
\hline \multicolumn{4}{|l|}{ Farley $^{d}$} \\
\hline $1-3$ & 2,7 & 28,4 & 28,4 \\
\hline $4-6$ & 5,4 & 29,8 & 14,5 \\
\hline $7-9$ & 13,1 & 44,6 & 5,8 \\
\hline $10-12$ & 39,0 & 36,4 & 3,9 \\
\hline \multicolumn{4}{|l|}{ Gebiet } \\
\hline Hamburg-Harvestehude & 8,7 & 32,7 & 10,3 \\
\hline Hamburg-Bergedorf & 11,2 & 34,3 & 17,2 \\
\hline Köln-Ostheim & 6,3 & 29,5 & 13,7 \\
\hline Köln-Rondorf & 6,7 & 24,8 & 17,4 \\
\hline Mülheim-Mitte & 12,8 & 27,3 & 14,5 \\
\hline Mülheim-Saarn & 7,9 & 24,1 & 17,0 \\
\hline
\end{tabular}

${ }^{a}$ Restliche Typen: T3: 1x uneingeschränkt, 2x beschränkt; T4: alle beschränkt; T5: 2x uneingeschränkt, 1x völlig unterbunden; T7: alle

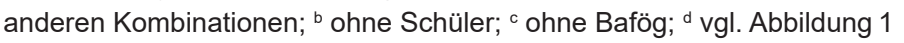

damit möglicherweise dazu gezwungen werden, ihre Vorurteile abzubauen: „Es ist wichtig, dass in unsere behütende Wohnumgebung (Disneyland) auch die Realität Einzug hält. Auch ein Wohngebiet wie Harvestehude muss seinen Anteil leisten“ (H-Harv. 596). „Gut, dass es einzelne Wohnungen sind anstatt Heime, dadurch kann Integration gefördert werden" (M-Mitte 4097).

Unsere Hypothese 2.1., die Befragten würden die Flüchtlingsunterkünfte in ihren Wohngebieten ablehnen, kann nicht bestätigt werden. Die positive Einstellung steigt von Mülheim-Saarn und Mülheim-Mitte zu KölnOstheim und Hamburg-Bergedorf bis zu Köln-Rondorf und Hamburg-Harvestehude. Die Unsicherheit und Angst vor Kriminalität sinkt auch signifikant $(p<.001)$, je gebildeter die Befragten sind und desto höhere Einkommen sie haben.

Ferner haben wir vermutet, dass auch Personen, die den Flüchtlingen positiv gegenüberstehen, die Flüchtlingsunterkunft im eigenen Wohngebiet ablehnen könnten. Um dies zu prüfen, haben wir die offenen 
Fragen zu den Flüchtlingen und der Unterkunft trichotomisiert in positiv, neutral und negativ. Bezogen auf alle Befragten beurteilen 20,4\% sowohl die Flüchtlinge als auch deren Unterkunft positiv. Dagegen sind es nur $5,3 \%$, welche die Flüchtlinge positiv, aber die Unterkunft negativ bewerten. Weitere $16,2 \%$ bewerten beide negativ. Der hochsignifikante Zusammenhang zwischen Einstellungen zu Flüchtlingen und zur Flüchtlingsunterkunft $(p<.001)$ belegt die vermutete enge Beziehung. Allerdings ist unsere Hypothese 2.2. widerlegt, die Einstellungen konfligieren nicht, sie korrespondieren.

\section{4 Ängste und Vorurteile}

Die Gebiete unterscheiden sich in allen Merkmalen beträchtlich (Angst-Skala, vgl. Tabelle 6). So machen die Terrorgefahr und die Konkurrenz auf dem Arbeitsmarkt den Befragten in Hamburg-Harvestehude am seltensten, in Mülheim-Mitte dagegen am häufigsten Angst. Dagegen ist die Furcht vor der Konkurrenz auf dem Wohnungsmarkt in den beiden Kölner Wohngebieten am höchsten.

Auf die Frage des Farley-Verfahrens, bis zu welchem Anteil grau gefärbter Häuser man bereit wäre, in dem Gebiet zu wohnen, gaben die meisten Befragten an, einen Flüchtlingsanteil zwischen 33 und $41 \%$ im Wohngebiet tolerieren zu können. Im ALLBUS 2016 ist der häufigste Wert 6 aus 49, das entspricht einem Anteil von nur 12,2 \%. Auffällig ist, dass ein Drittel der Befragten nicht in einem Wohngebiet mit wenigen Flüchtlingen (Gebiete bis 12 \% Flüchtlingsanteil) wohnen möchten, weil das „langweilig“ oder „zu wenig gemischt“ sei.

Die Angst-Skala korreliert mit $r=.44$ mit dem FarleyVerfahren. Je mehr Ängste eine Person mit der Zuwanderung von Flüchtlingen verbindet, desto weniger Flüchtlinge akzeptiert sie in ihrem Wohngebiet. Dies entspricht unserer Hypothese 2.3.

\subsection{Kontakte und Vorurteile}

Folgt man der Kontakt-Hypothese, so sollten Kontakte zu Ausländern oder Migranten zu positiveren Einstellungen gegenüber Migranten führen; hier: zu Flüchtlingen. Ob die Befragten Kontakte zu Flüchtlingen haben, bejahten in Hamburg-Harvestehude $24,7 \%$, in Hamburg-Bergedorf 29,8 \%, in Köln-Ostheim 24,2 \%, in Köln-Rondorf 28,5 \%, in Mülheim-Mitte 33,1 \% und in Mülheim-Saarn 22,3\%. Demnach haben immerhin bis zu ein Drittel der Befragten Kontakte zu Flüchtlingen. Ein großer Teil der Befragten mit Kontakt hat diesen gezielt hergestellt und engagiert sich für Flüchtlinge. Insofern bestehen gemeinsame Ziele, die kontaktfördernd sind. Schließlich können wir von einer institutionellen - nationalen - Unterstützung ausgehen, die mit dem Ausdruck "Willkommenskultur" einerseits und der großen Zahl von ehrenamtlich Tätigen andererseits, belebt wird.

Wenn nun die Kontakte Vorurteile verringern, dann sollte es signifikante Beziehungen zur Angst-Skala und zum Farley-Verfahren geben. Da die Kontakte dichotom gemessen wurden, haben wir als Testverfahren Varianzanalysen (ANOVA) berechnet. Die Ergebnisse entsprechen unseren Annahmen: Für Kontakte vs. AngstSkala erhalten wir einen Wert von $F=32,43$, für Kontakte vs. Farley-Verfahren ist $\mathrm{F}=68,35$ (beide $p<.001$ ). Kontakte wirken sich demnach hochsignifikant positiv aus (obgleich wir mit den Querschnittsdaten keinen Effekt behaupten können). Unsere Ergebnisse zu Kontakten stützen auch vorhandene Befunde aus der Literatur (vgl. Kapitel 2.2): Personen höheren Alters haben weniger Kontakte zu Flüchtlingen, hingegen steigen die Kontakte mit steigender Schulbildung. Unterschiede in den Kontakten nach dem Geschlecht konnten wir nicht feststellen.

Eine interessante Erweiterung der Kontakt-Theorie ist von Hewstone (2004) vorgeschlagen worden: Auch indirekte Kontakte einer Person (= Ich weiß, Mitglieder meiner Gruppe haben Kontakte zu Mitgliedern der Fremdgruppe) können dazu führen, bei dieser Person die Vorurteile zu verringern. Das gilt für die von uns Befragten: Indirekte Kontakte (gemessen über Kontakte in der Familie) führen dazu, mehr Flüchtlinge im Wohngebiet zu akzeptieren (ANOVA, $F=10,044, p<.001$ ). Die Kontakt-Hypothese wird somit weitgehend durch die Analysen gestützt (H3).

\section{Zusammenfassende Analyse}

Unsere Studie zeigt, wie unterschiedlich die Bewohner der sechs Gebiete auf die Flüchtlinge und die Unterkünfte reagieren. Dabei müssten wir mögliche Gebiets(Kontext-)Effekte von den Individualeffekten trennen. Das wäre mit einer Mehrebenenanalyse möglich, doch dazu wären mindestens zwölf Gebiete erforderlich. Wir können also nicht messen, ob allein die soziodemographische Zusammensetzung des Gebiets (Kompositionseffekt) oder auch Gebietsmerkmale die Einstellungen der Bewohner beeinflussen.

Um nun die unterschiedlichen Befunde zusammenzubringen und dabei ansatzweise Kompositions- von 
Tabelle 6: Angst-Skala, Antworten „stimme voll zu“, nach Wohngebieten, in Prozent

\begin{tabular}{|c|c|c|c|c|c|c|}
\hline \multirow{2}{*}{$\begin{array}{l}\text { Merkmal } \\
\text { Ich befürchte, dass ... }\end{array}$} & \multicolumn{2}{|l|}{ Hamburg } & \multicolumn{2}{|l|}{ Köln } & \multicolumn{2}{|c|}{ Mülheim an der Ruhr } \\
\hline & $\begin{array}{l}\text { Harveste- } \\
\text { hude }\end{array}$ & Bergedorf & Ostheim & Rondorf & Mitte & Saarn \\
\hline $\begin{array}{l}\text { 1. die Verschuldung der } \\
\text { öffentlichen Haushalte } \\
\text { zunimmt }\end{array}$ & 16,1 & 17,4 & 24,2 & 23,6 & 22,5 & 24,2 \\
\hline $\begin{array}{l}\text { 2. die Konkurrenz auf dem } \\
\text { Wohnungsmarkt größer wird }\end{array}$ & 20,9 & 32,9 & 41,5 & 41,4 & 31,7 & 23,5 \\
\hline $\begin{array}{l}\text { 3. die Kosten für die Unter- } \\
\text { bringung und Versorgung zu } \\
\text { hoch sind }\end{array}$ & 10,9 & 21,0 & 25,3 & 22,1 & 22,7 & 22,0 \\
\hline $\begin{array}{l}\text { 4. der Einfluss des Islam in } \\
\text { Deutschland zu stark wird }\end{array}$ & 14,2 & 21,7 & 24,8 & 32,8 & 32,9 & 30,5 \\
\hline $\begin{array}{l}\text { 5. die Zahl der Straftaten } \\
\text { zunimmt }\end{array}$ & 12,7 & 21,4 & 25,8 & 22,5 & 29,8 & 19,5 \\
\hline $\begin{array}{l}\text { 6. die Terrorgefahr in } \\
\text { Deutschland steigt }\end{array}$ & 12,5 & 25,7 & 29,4 & 29,8 & 35,7 & 29,6 \\
\hline $\begin{array}{l}\text { 7. der Einfluss fremder } \\
\text { Kulturen in Deutschland } \\
\text { zunimmt }\end{array}$ & 8,1 & 22,8 & 24,0 & 23,8 & 26,9 & 27,7 \\
\hline $\begin{array}{l}\text { 8. der Wohlstand in } \\
\text { Deutschland bedroht wird }\end{array}$ & 4,7 & 6,9 & 9,8 & 8,3 & 13,9 & 8,5 \\
\hline $\begin{array}{l}\text { 9. die Konkurrenz am } \\
\text { Arbeitsmarkt größer wird }\end{array}$ & 7,3 & 12,8 & 13,4 & 14,8 & 22,7 & 15,4 \\
\hline $\begin{array}{l}\text { 10. die Sicherheit im öffentli- } \\
\text { chen Raum geringer wird }\end{array}$ & $-10,9$ & 15,3 & 21,4 & 19,4 & 25,8 & 23,7 \\
\hline $\begin{array}{l}\text { 11. unsere freizügige } \\
\text { Lebensweise nicht mehr } \\
\text { möglich wird }\end{array}$ & 5,8 & 8,9 & 11,6 & 10,0 & 13,0 & 12,5 \\
\hline
\end{tabular}

Signifikanzen Wohngebiete:

1: $\mathrm{Chi}^{2}=42,07, \mathrm{p}<.001 ; 2: \mathrm{Chi}^{2}=64,90, \mathrm{p}<.001 ; 3: \mathrm{Chi}^{2}=44,39, \mathrm{p}<.001$;

4: $\mathrm{Chi}^{2}=50,25, \mathrm{p}<.001 ; 5: \mathrm{Chi}^{2}=52,87, \mathrm{p}<.001 ; 6: \mathrm{Chi}^{2}=57,38, \mathrm{p}<.001$;

7: $\mathrm{Chi}^{2}=75,90, \mathrm{p}<.001 ; 8: \mathrm{Chi}^{2}=58,55, \mathrm{p}<.001 ; 9: \mathrm{Chi}^{2}=42,48, \mathrm{p}<.001$;

10: $\mathrm{Chi}^{2}=47,97, \mathrm{p}<.001 ; 11: \mathrm{Chi}^{2}=33,96, \mathrm{p}<.003$.

Individualeffekten zu unterscheiden, haben wir das Verfahren der Multiplen Korrespondenzanalyse (MCA) gewählt. Es handelt sich dabei um ein exploratives multivariates Verfahren für die grafische und numerische Analyse nahezu aller Datentypen (Blasius/Greenacre 2006: 4). Die Interpretation der Ergebnisse erfolgt hierbei über die grafische Verortung der Variablen in einem mehrdimensionalen Raum (Blasius 2010; Le Roux/
Rouanet 2010). Häufig geschieht dies in Verbindung mit der Theorie des sozialen Raums nach Bourdieu (2014). Liegen zwei Variablen im Koordinatensystem nah beieinander, so wurden sie häufig von Personen mit einem ähnlichen sozialen Status zusammen genannt. Liegen Variablen weit auseinander, sind die Antworten dagegen selten in Kombination angeführt worden. Das Ergebnis zeigt Abbildung 2. 
Der soziale Raum wird durch zwei Achsen strukturiert, die sich als kulturelles und ökonomisches Kapital bezeichnen lassen. Dabei ordnet sich das ökonomische Kapital besonders nach der Frage, ob die Person in einer Eigentumswohnung, einer Mietwohnung oder einer Sozialwohnung wohnt. Gleichfalls bewahren auch die gruppierten Äquivalenzeinkommen ihre Ordinalität, wobei sich die Deutung sowohl auf das monatliche Äquivalenzeinkommen des Haushalts als auch auf bestehende Besitzverhältnisse im Hinblick auf den eigenen Wohnraum beziehen lässt.

Die sechs Gebiete verteilen sich deutlich über die vier Quadranten. Wir haben - absichtlich - sehr verschiedene Gebiete untersucht. Hamburg-Harvestehude und Köln-Ostheim bilden die Gegensätze im kulturellen Kapital, Köln-Rondorf und Mülheim-Mitte im ökonomischen Kapital. Auf der ökonomischen Achse verteilen sich auch Wohneigentum, Miete und Sozialmieter wie erwartet.

Hamburg-Harvestehude weist nicht nur die höchste Bildung auf, es liegt räumlich auch nah an den Werten der höchsten Akzeptanz von Flüchtlingen im Wohngebiet (Far 10-12). Hamburg-Bergedorf hat ebenfalls viele Bewohner mit Abitur und Kontakten zu Flüchtlingen (Kontakt FI_Ja). Das soziale Kapital (kollektive Effektivität) ist mittel bis hoch (Coll_Eff_3,4). Dem ökonomischen Kapital folgend kämen dann Köln-Rondorf und Mülheim-Saarn, wobei Köln-Rondorf das höhere ökonomische Kapital aufweist. Dort wohnen auch mehr Eigentümer. Das soziale Kapital ist hoch, das kulturelle durchschnittlich. Die Bewohner sind älter. Die Toleranz ist eher gering.

Das Wohngebiet Mülheim-Mitte zeichnet sich durch ein geringes ökonomisches und ein durchschnittliches kulturelles Kapital aus. Es sind eher jüngere Befragte. Es gibt kaum Kontakte zu Flüchtlingen und das soziale Kapital ist gering (Coll_Eff_1, 2). Die Akzeptanz liegt im mittleren Bereich. Die Bewohner von Köln-Ostheim schließlich haben ein niedriges kulturelles und ein mittleres bis geringes ökonomisches Kapital. Auch das soziale Kapital ist niedrig und die Akzeptanz von Flüchtlingen im Wohngebiet gering.

Bezüglich unserer Annahme zum sozialen Kapital $(\mathrm{H} 4)$ zeigt sich ein auffälliges Ergebnis: Der Zusammenhang ist kurvilinear, nicht linear. Die Akzeptanz steigt bis zu einem Wert von Coll_Eff $=4$ an, nimmt jedoch ab Coll_ $\mathrm{Eff}=5$ wieder $\mathrm{ab}$. Die Hypothese kann daher nur teilweise bestätigt werden.

Die Korrespondenzanalyse zeigt auch, dass die Annahme H5, die Unterschiede zwischen den Gebieten seien geringer als die Unterschiede zwischen den
Städten, nicht zutrifft. Vielmehr unterscheiden sich die Gebiete erheblich, vorrangig aufgrund ihrer Sozialstruktur.

\section{Zusammenfassung und Folgerungen}

Wir haben die Einstellungen zu Flüchtlingen in sechs Wohngebieten mit unterschiedlichem sozialen Status in Hamburg, Köln und Mülheim an der Ruhr untersucht, nachdem dort Flüchtlinge untergebracht worden waren Die Anwohner zeigen trotz anfänglicher Proteste, z. B. in Hamburg-Harvestehude, eine weitgehend positive Einstellung zu der Flüchtlingsunterkunft und allgemein zu Flüchtlingen. Beide Einstellungen korrelieren signifikant positiv: Befragte, die positiv über Flüchtlinge denken, befürworten auch eher die Flüchtlingsunterkunft. Die wichtigste Determinante der Akzeptanz ist die Schulbildung (oder das kulturelle Kapital) (Leßke/Friedrichs/ Schwarzenberg 2018: 232). Es lassen sich aber auch erhebliche Unterschiede in den Einstellungen nach soziodemographischen Merkmalen belegen. So zeigt sich, dass das Geschlecht keinen Einfluss auf die Einstellung gegenüber Flüchtlingen hat. Hingegen nehmen mit zunehmendem Alter der Befragten die Vorurteile zu; dies entspricht den Befunden von Hafez und Schmidt (2015). Mit steigender Schulbildung, in etwas geringerem Maße mit steigendem Einkommen, nehmen die Vorurteile ab (vgl. unter anderem Zick/Küpper/Hövermann 2011). Die Gründe hierfür sind eine geringere wirtschaftliche und soziale Konkurrenz sowie vermutlich ein breiteres Wissen über die Minorität.

Ein erheblicher Teil der Befragten verbindet mit dem Zuzug von Flüchtlingen zahlreiche Ängste, die wir mit einer Skala gemessen haben. Je größer diese Ängste sind, desto größer ist auch die Ablehnung von Flüchtlingen im Wohngebiet.

Damit bewähren sich fast alle von uns auf der Basis der Theorien von Allport und Pettigrew formulierten Hypothesen H1.1., H1.2., H1.3., H2.3. und H3. Zudem finden wir auch die von Hewstone postulierten positiven Wirkungen indirekter Kontakte. Hypothese 4 kann teilweise bestätigt werden. Einzig die Hypothesen - H2.1. und H2.2. -, die besagten, dass Anwohner eine negative Einstellung gegenüber einer in ihrem Wohngebiet errichteten Unterkunft hätten, sowie H5 müssen abgelehnt werden.

Die Ergebnisse sprechen deutlich dafür, dass es nicht gleichgültig ist, in welchem Wohngebiet man eine 


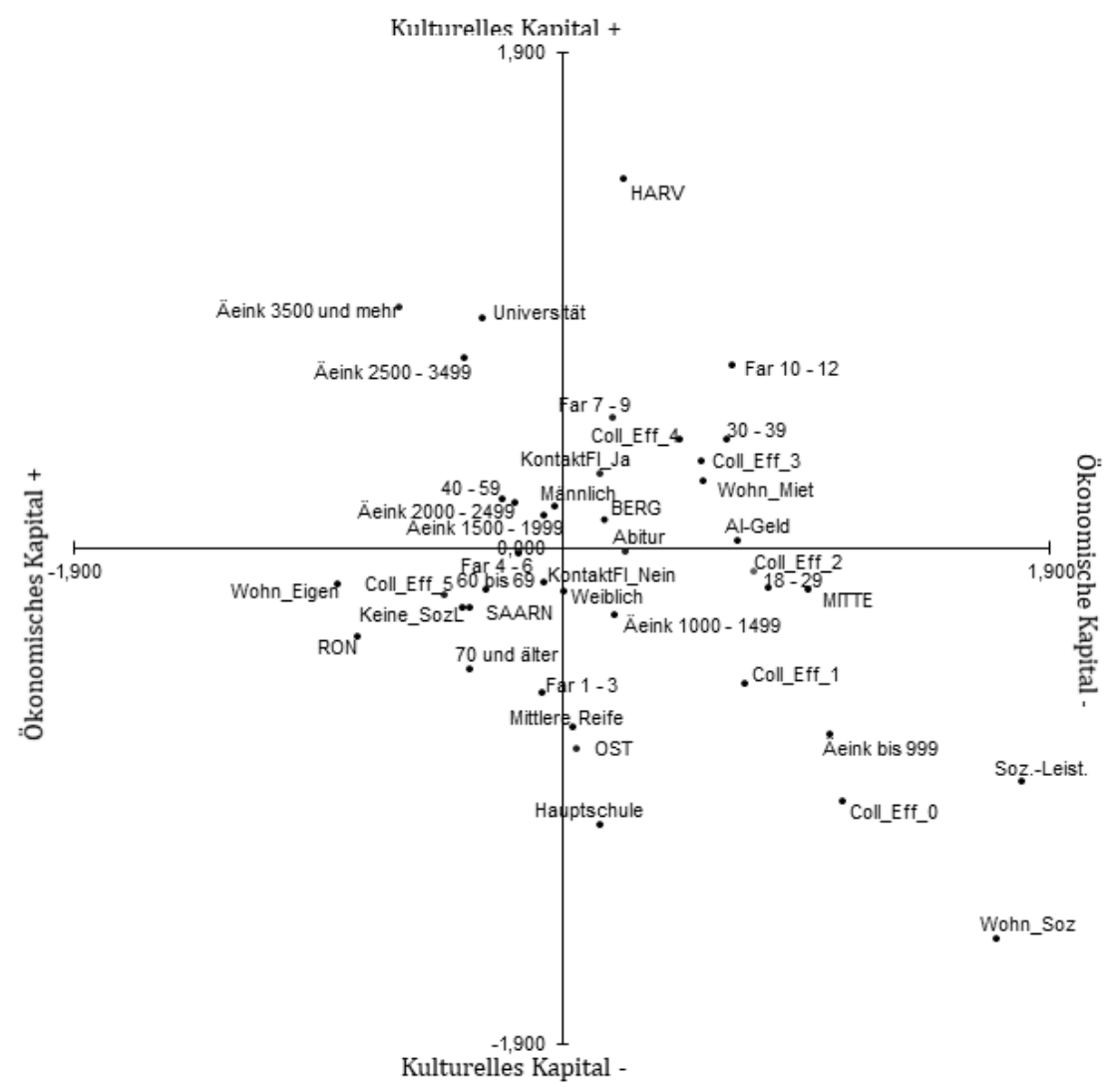

Abbildung 2: Korrespondenzanalyse

Flüchtlingsunterkunft errichtet. Wir vermuten, wie ansatzweise in der Korrespondenzanalyse nachgewiesen, dass es neben dem Kompositions- auch einen Gebietseffekt gibt. Ein Wohngebiet wie Hamburg-Harvestehude ist demnach besonders gut geeignet für eine Flüchtlingsunterkunft. Ähnlich gut geeignet erscheint uns HamburgBergedorf, allerdings ist hier der Anteil der Anwohner mit hoher Schulbildung niedriger. Mülheim-Saarn und KölnRondorf wirken aufgrund des nachgewiesenen hohen sozialen Kapitals vertretbar für eine Flüchtlingsunterkunft, wenngleich aufgrund der hohen Eigentumsquote und dem größeren Anteil an älteren Personen stärkere Vorbehalte bestehen. In einem Wohngebiet wie KölnOstheim hingegen zeigten sich zwar weniger öffentliche Proteste, dafür mehr Ablehnung. Daher sind Gebiete mit einem niedrigen kulturellen Kapital, einem hohen Anteil mit Personen mit niedrigen Einkommen und einer niedrigen kollektiven Effektivität wenig geeignet.

Ein besonderer Fall ist Mülheim-Mitte. Hier sind die Flüchtlinge nicht in einer Unterkunft, sondern in Wohnungen, verteilt über das Wohngebiet, untergebracht. Diese Lösung wird von der überwiegenden Zahl der Befragten positiv beurteilt, dies entspricht den Erkenntnissen von Aumüller, Daphi und Biesenkamp (2015). Negative Einstellungen gibt es dennoch, unter anderem weil es sich um ein statusniedriges Wohngebiet mit einem hohen Anteil von Migranten und einem niedrigen Bildungsniveau handelt.

Weitere Analysen zur Akzeptanz von Flüchtlingen und Flüchtlingsunterkünften könnten sich auf Ostdeutschland und kleinere Städte dort fokussieren. Sie sollten dabei insbesondere mit multivariaten Verfahren die Effekte der Statusunterschiede der Bewohner auf die Einstellungen und die Unterscheidung von Einstellungen zu Flüchtlingen und zum Islam behandeln. Des Weiteren sind die Einstellungen von Personen mit Migrationshintergrund gegenüber Flüchtlingen zu untersuchen.

Danksagung: Wir danken der Fritz Thyssen Stiftung (Az. 20.15.0.088SO), der Körber Stiftung und dem Forschungsinstitut für gesellschaftliche Weiterentwicklung (FGW) (Förderkennzeichen: 005-1605-0042) sehr für die Förderung des Projekts. Wir danken auch Dr. 
Pamela Kerschke-Risch dafür, dass sie die Feldphase in Hamburg organisiert hat.

\section{Literatur}

Ahrens, P.-A. (2017): Skepsis und Zuversicht. Wie blickt Deutschland auf Flüchtlinge? Erwartungen der Bevölkerung zur Aufnahme von Flüchtlingen zwischen November 2015 und April 2017. Hannover.

Allport, G. W. (1954): The nature of prejudice. Cambridge.

Asbrock, F.; Kauff, M.; Issmer, C.; Christ, O.; Pettigrew, T.F.; Wagner, U. (2012): Kontakt hilft - auch wenn die Politik es nicht immer leichtmacht. In: Heitmeyer, W. (Hrsg.): Deutsche Zustände. Folge 10. Berlin, 199-219.

Aumüller, J. (2018): Die kommunale Integration von Flüchtlingen. In: Gesemann, F.; Roth, R. (Hrsg.): Handbuch Lokale Integrationspolitik. Wiesbaden, 173-198. doi: 10.1007/978-3-65813409-9

Aumüller, J.; Daphi, P.; Biesenkamp, C. (2015): Die Aufnahme von Flüchtlingen in den Bundesländern und Kommunen. Behördliche Praxis und zivilgesellschaftliches Engagement. Stuttgart.

Bauer, I. (2017): Unterbringung von Flüchtlingen in deutschen Kommunen. Konfliktmediation und lokale Beteiligung. Osnabrück/ Bonn. = State-of-Research Papier 10, Verbundprojekt ,Flucht: Forschung und Transfer'.

Baur, H.; Klein, D.; Seuring, J.; Walcher, G.; Weidner, A. (2010): Fremdenfeindlichkeit im Ost-Westdeutschen Vergleich. Welchen Erklärungsbeitrag leisten Kontakt- und Konflikthypothese? In: Siegert, M.; Kogan, I. (Hrsg.): Einstellungen gegenüber ethnischen Minderheiten in Europa. Analysen mit dem European Social Survey. Bamberg, 1-34. = Bamberger Beiträge zur Soziologie 6.

Billiet, J.; Meuleman, B.; de Witte, H. (2014): The relationship between ethnic threat and economic insecurity in times of economic crisis. Analysis of European Social Survey Data. In: Migration Studies 2, 2, 135-161. doi: 10.1093/migration/mnu023

Blalock, H. M. (1967): Toward a Theory of Minority Group Relations. New York.

Blasius, J. (2010): Korrespondenzanalyse. In: Wolf, C.; Best, H. (Hrsg.): Handbuch der sozialwissenschaftlichen Datenanalyse. Wiesbaden, 367-389. doi: 10.1007/978-3-531-92038-2

Blasius, J.; Friedrichs, J. (2007): Internal Heterogeneity of a Deprived Urban Area and its Impact on Residents' Perception of Deviance. In: Housing Studies 22, 5, 753-780. doi: 10.1080/02673030701474693.

Blasius, J.; Friedrichs, J.; Klöckner, J. (2008): Doppelt benachteiligt? Leben in einem deutsch-türkischen Stadtteil. Wiesbaden. doi: 10.1007/978-3-531-91176-2

Blasius, J.; Greenacre, M. J. (2006): Correspondence Analysis and Related Methods in Practice. In: Greenacre, M. J.; Blasius, J. (Hrsg.): Multiple Correspondence Analysis and Related Methods. Boca Raton, 3-40.

Bourdieu, P. (2014): Die feinen Unterschiede. Kritik der gesellschaftlichen Urteilskraft. Frankfurt am Main.

Brücker, H.; Rother, N.; Schupp, J. (2016): IAB-BAMF-SOEP-Befragung von Geflüchteten. Überblick und erste Ergebnisse. Nürn- berg. = Bundesamt für Migration und Flüchtlinge Forschungsbericht 29.

Dixon, J. C. (2006): The Ties That Bind and Those That Don't. Toward Reconciling Group Threat and Contact Theories of Prejudice. In: Social Forces 84, 4, 2179-2204.

Dymarz, M.; Hanhörster, H.; Hans, N.; Wallraff, M.; Zimmer-Hegmann, R. (2016): Gelingende Integration im Quartier. Gutachten des ILS - Institut für Landes- und Stadtentwicklungsforschung. Dortmund.

Ellison, C. G.; Shin, H.; Leal, D. L. (2011): The contact hypothesis and attitudes toward Latinos in the United States. In: Social Science Quarterly 92, 4, 938-958. doi: 10.1111/j.15406237.2011.00798.x

Farley, R.; Schuman, H.; Bianchi, S.; Colasanto, D.; Hatchett, S. (1978): "Chocolate City, Vanilla Suburbs". Will the Trend toward Racially Separate Communities Continue? In: Social Science Research 7, 4, 319-344. doi: 10.1016/0049-089X(78)90017-0

Farwick, A. (2009): Segregation und Eingliederung. Zum Einfluss der räumlichen Konzentration von Zuwanderern auf den Eingliederungsprozess. Wiesbaden. doi: 10.1007/978-3-53191369-8

Fetzer, J. S. (2000): Public attitudes toward immigration in the United States, France, and Germany. Cambridge.

Friedrichs, J.; Leßke, F.; Schwarzenberg, V. (2017): Sozialräumliche Integration von Flüchtlingen. Das Beispiel Hamburg-Harvestehude. In: Aus Politik und Zeitgeschichte 67, 27-29, 34-40.

Friedrichs, J.; Leßke, F.; Schwarzenberg, V. (2019): Fremde Nachbarn. Die sozialräumliche Integration von Flüchtlingen in Hamburg, Köln und Mülheim an der Ruhr. Wiesbaden (in Vorbereitung).

GESIS - Leibniz Institut für Sozialwissenschaften (2011): ALLBUS/ GGSS 2006 (Allgemeine Bevölkerungsumfrage der Sozialwissenschaften/German General Social Survey 2006). Köln.

GESIS - Leibniz-Institut für Sozialwissenschaften (2017): Allgemeine Bevölkerungsumfrage der Sozialwissenschaften ALLBUS 2016. Köln

Grote, J. (2018): Die veränderte Fluchtmigration in den Jahren 2014 bis 2016. Reaktionen und Maßnahmen in Deutschland. Nürnberg. = Working Paper 79 des Forschungszentrums des Bundesamtes für Migration und Flüchtlinge.

Hafez, K.; Schmidt, S. (2015): Die Wahrnehmung des Islams in Deutschland. Gütersloh.

Hewstone, M. (2004): Neuere Forschungen über Intergruppenkonflikte. Konsequenzen für den Umgang mit Migration und Integration. Berlin. = WZB Discussion Papers 2004-601.

Infratest-dimap (2016): Umfrage zum Thema „Flüchtlinge/Integration“ im Auftrag des NDR. Berlin.

Jacobsen, J.; Eisnecker, P.; Schupp, J. (2017): In 2016, around one-third of people in Germany donated for refugees and ten percent helped out on site - yet concerns are mounting. In: DIW Economic Bulletin 16+17.2017, 165-177.

Le Roux, B.; Rouanet, H. (2010): Multiple Correspondence Analysis. Los Angeles.

Leibold, J.; Thörner, S.; Gosen, S.; Schmidt, P. (2012): Mehr oder weniger erwünscht? Entwicklung und Akzeptanz von Vorurteilen gegenüber Muslimen und Juden. In: Heitmeyer, W. (Hrsg.): Deutsche Zustände. Folge 10. Berlin, 177-198.

Leßke, F.; Friedrichs, J.; Schwarzenberg, V. (2018): Die Klassifizierung von Flüchtlingen im sozialen Raum. Eine empirische Analyse relationaler Beziehungen in der Flüchtlingsmigration. 
In: Tewes, O.; Gül, G. (Hrsg.): Der soziale Raum der postmigrantischen Gesellschaft. Weinheim, 217-239.

Lubbers, M.; Coenders, M.; Scheepers, P. (2006): Objections to Asylum Seeker Centres. Individual and Contextual Determinants of Resistance to Small and Large Centres in the Netherlands. In: European Sociological Review 22, 3, 243-257. doi: 10.1093/esr/jci055

Mansel, J. (2006): Emotionale Verarbeitung der Interaktionen mit Zuwanderern und fremdenfeindliche Einstellungen. In: Soziale Probleme. Zeitschrift für soziale Probleme und soziale Kontrolle 17, 1, 90-114.

Martinović, B. (2013): The Inter-Ethnic Contacts of Immigrants and Natives in the Netherlands. A Two-Sided Perspective. In: Journal of Ethnic and Migration Studies 39, 1, 69-85. doi: 10.1080/1369183X.2013.723249

Martinović, B.; van Tubergen, F.; Maas, I. (2009): Dynamics of Interethnic Contact. A Panel Study of Immigrants in the Netherlands. In: European Sociological Review 25, 3, 303-318.

Mayring, P. (2010): Qualitative Inhaltsanalyse. Grundlagen und Techniken. Weinheim.

Michaelis, S. (2016): Wann wird aus räumlicher Nähe Kontakt? Einstellungen von Anwohnern Kölner Flüchtlingsunterkünfte zu Flüchtlingen. Bachelorarbeit an der Universität zu Köln.

Mühlenfeld, D. (2015): Flüchtlinge in Mülheim an der Ruhr. Unterbringung, Versorgung und Begleitung vernetzt gestalten. In: Stadt und Gemeinde 70, 4, 128-130.

Oberwittler, D. (2004): Stadtstruktur, Freundeskreise und Delinquenz. Eine Mehrebenenanalyse zu sozialökologischen Kontexteffekten auf schwere Jugenddelinquenz. In: Oberwittler, D.; Karstedt, S. (Hrsg.): Soziologie der Kriminalität. Wiesbaden, 135-170. = Kölner Zeitschrift für Soziologie und Sozialpsychologie, Sonderheft 43.

Petermann, S.; Schönwälder, K. (2014): Immigration and social interaction. In: European Societies 16, 4, 500-521. doi: 10.1080/14616696.2013.865064

Pettigrew, T. F. (1998): Intergroup Contact Theory. In: Annual Review of Psychology 49, 65-85. doi: 10.1146/annurev.psych.49.1.65

Pettigrew, T. F.; Tropp, L. R. (2000): Does intergroup contact reduce prejudice? Recent meta-analytic findings. In: Oskamp, S. (Hrsg.): Reducing prejudice and discrimination. Mahwah, NJ, 93-114.

Pettigrew, T. F.; Tropp, L. R. (2006): A Meta-Analytic Test of Intergroup Contact Theory. In: Journal of Personality and Social Psychology 90, 5, 751-783. doi: 10.1037/0022-3514.90.5.751

Pettigrew, T. F.; Tropp, L. R. (2011): When Groups Meet: The Dynamics of Intergroup Contact. New York.

Quillian, L. (1995): Prejudice as a Response to Perceived Group Threat. Population Composition and Anti-Immigrant and Racial Prejudice in Europe. In: American Sociological Review 60, 4, 586-611. doi: 10.2307/2096296

Quillian, L. (1996): Group Threat and Regional Change in Attitudes toward African-Americans. In: American Journal of Sociology 102, 3, 816-860.

Sampson, R.J. (2012): Great American City. Chicago and the Enduring Neighborhood Effect. Chicago, IL.

Sampson, R.J.; Groves, W.B. (1989): Community Structure and Crime: Testing Social-Disorganization Theory. In: American Journal of Sociology 94, 4, 774-802.
Sampson, R.J.; Raudenbush, S.W.; Earls, F. (1997): Neighborhoods and Violent Crime. A Multilevel Study of Collective Efficacy. In: Science 277, 5328, 918-924. doi: 10.1126/science.277.5328.918

Scheepers, P.; Gijsberts, M.; Coenders, M. (2002): Ethnic Exclusionism in European Countries. Public Opposition to Civil Rights for Legal Migrants as a Response to Perceived Ethnic Threat. In: European Sociological Review 18, 1, 17-34.

Schelling, T.C. (1971): Dynamic models of segregation. In: Journal of Mathematical Sociology 1, 2, 143-186. doi: 10.1080/0022250X.1971.9989794

Schlueter, E.; Scheepers, P. (2010): The relationship between outgroup size and anti-outgroup attitudes. A theoretical synthesis and empirical test of group threat- and intergroup contact theory. In: Social Science Research 39, 2, 285-295. doi: 10.1016/j.ssresearch.2009.07.006

Schmid, K.; Al Ramiah, A.; Hewstone, M. (2014): Neighborhood ethnic diversity and trust: The role of intergroup contact and perceived threat. In: Psychological Science 25, 3, 665-674. doi: 10.1177/0956797613508956

Schneider, S. L. (2008): Anti-Immigrant Attitudes in Europe. Outgroup Size and Perceived Ethnic Threat. In: European Sociological Review 24, 1, 53-67. doi: 10.1093/esr/jcm034

Semyonov, M.; Raijman, R.; Anat, Y. T.; Schmidt, P. (2004): Population size, perceived threat and exclusion. A multiple-indicators analysis of attitudes toward foreigners in Germany. In: Social Science Research 33, 4, 681-701. doi: 10.1016/j.ssresearch.2003.11.003

Smith, C. B. (1994): Back and to the Future. The Intergroup Contact Hypothesis Revisited. In: Sociological Inquiry 64, 4, 438-455. doi: 10.1111/j.1475-682X.1994.tb00401.x

Stephan, W. G.; Ybarra, O.; Morrison, K. R. (2009): Intergroup threat theory. In: Nelson, T. D. (Hrsg.): Handbook of Prejudice, Stereotyping, and Discrimination. New York, 43-59.

Taylor, M. C. (1998): How white attitudes vary with the racial composition of local populations: numbers count. In: American Sociological Review 63, 4, 512-535. doi: 10.2307/2657265

Taylor, M. C. (2000): The significance of racial context. In: Sears, D. O.; Sidanius, J.; Bobo, L. (Hrsg.): Racialized Politics. The Debate about Racism in America. Chicago, 118-136.

von Gostomski, B.; Stichs, C.; Stichs, A. (2008): Der Einfluss von Gelegenheitsstrukturen auf die Häufigkeit des Kontakts von Zuwanderern mit Deutschen. In: Hillmann, F.; Windzio, M. (Hrsg.): Migration und städtischer Raum. Chancen und Risiken der Segregation und Integration. Opladen/Farmington Hills, 279-296.

Wagner, U.; Christ, O.; Kühnel, S. (2003): Diskriminierendes Verhalten. Es beginnt mit Abwertung. In: Heitmeyer, W. (Hrsg.): Deutsche Zustände. Folge 2. Frankfurt am Main, 110-122.

Zick, A.; Küpper, B.; Hövermann, A. (2011): Intolerance, Prejudice and Discrimination. A European Report. Bonn. 